(From the Laboratory of the Livermore Sanatorium, California, U.S.A.)

\title{
Über \\ die Analogie zwischen der Wasserabsorption durch Fibrin und durch Muskel.
}

Von

Martin H. Fischer.

(Mit 13 Textfiguren.)

\section{Einleitung.}

Im folgenden soll über Versuche, die bis jetzt nur präliminarisch veröffentlicht worden sind ${ }^{1}$ ), ausführlich berichtet werden. Die Versuche befassen sich mit der Absorption und Sekretion von Wasser durch Froschmuskeln unter verschiedenen äusseren Umständen und sollen die Analogie, die zwischen der Absorption von Wasser durch Fibrin und der Absorption von Wasser durch Muskel besteht, darlegen, um hierdurch einen Beweis, dass die Absorption und Sekretion von Wasser durch Tier- und Pflanzengewebe zum grossen Teil durch die veränderbare Affinität ${ }^{2}$ ) der Kolloide für Wasser bedingt ist, zu liefern ${ }^{3}$ ).

1) Martin H. Fischer und Gertrude Moore, American Jonrnal of Physiology vol. 20 p. 342. 1907.

2) Gegen den Ausdruck „veränderbare Affinität" können Einwendungen erhoben werden, denn er stellt keinen klaren physikalisch-chemischen Begriff dar. Doch ist das Wesen der Kräfte, die in der Absorption von Wasser durch Kolloide eine Rolle spielen, bis jetzt noch so unvollkommen erforscht, dass der Gebrauch eines allgemeinen Ausdruckes nötig ist. Zurzeit ist es nicht von Interesse, die verschieden an der Hand liegenden widersprechenden Theorien äber die Natur dieser Affinität von Kolloiden für Wasser zu diskutieren, sondern nur den Beweis zu erbringen, dass die Bewegung und Ansammlung von Wasser in Geweben Prozesse darstellen, die von den Ejgenschaften ihrer kolloiden Beschaffenheit im grossen Maasse abhängig sind.

3) Siehe Martin H. Fischer, Physiology of Alimentation p. 182, 187, 267 u. 268. New York 1907. 
Aus unseren Versuchen über die Quellung des Fibrins konnten Gertrude Moore und ich ${ }^{1}$ ) unter anderen die folgenden Schlussfolgerungen ziehen:

a) Fibrin quillt mehr in der Lösung irgendeiner Säure als in destilliertem Wasser, doch ist der Grad der Quellung grösser in manchen Säuren als in anderen.

b) Durch Zusatz irgendeines Salzes zu der reinen Säurelösung wird der Betrag des vom Fibrin absorbierten Wassers vermindert. Je höher der Prozentsatz des Salzes in der Säurelösung, desto weniger quillt das Fibrin in der Lösung. Chemisch äquivalente Mengen der verschiedenen Salze bringen nicht den gleichen Effekt hervor. Die Wirkung eines Salzes ist scheinbar durch die Summe der Wirkung seiner Ionen bestimmt, die augenscheinlich in bestimmt geordnete Reihen von Anionen und Kationen eingeteilt werden können.

c) Nachdem Fibrin in einer reinen Säurelösung ad maxima aufgequollen ist, quillt es noch mehr, wenn destilliertes Wasser für die Säure substituiert wird ${ }^{2}$ ).

d) Die Wasseraufnabme und -abgabe durch Fibrin stellen einen reversiblen Prozess dar. Doch ist der Prozess (innerhalb beschränkter Zeiträume) nicht vollständig umkehrbar.

e) Nicht-Elektrolyte teilen nicht mit Elektrolyten deren ausgesprochene Fähigkeit, die Wasserabsorption durch Fibrin in Säurelösungen $\mathrm{zu}$ beeinflussen ${ }^{3}$ ).

1) Martin H. Fischer und Gertrude Moore, American Journal of Physiology vol. 20 p. 330.1907.

2) Nach neueren Versuchen, die bald veröffentlicht werden sollen, ist dieser. Satz nur in dem Falle richtig, wenn die o p timale Konzentration, das heisst die Säurekonzentration, die die grösste Quellung des Fibrins hervorruft, überschritten worden ist.

3) Diese Quellungsfähigkeit gewisser Kolloide ermöglicht uns einen Begriff des Ursprunges der Wachstumsenergie. Die primäre Änderung während des Wachsens bildet eine erhöhte Wasserabsorption, deren Ursache man leicht darin finden kann, dass während des Wachșens Substanzen, die die Affinität der Kolloide für Wasser erhöhen (z. B. Säuren in den wachsenden Teilen der Pflanzen), gebildet werden, oder dass Kolloide mit einer nur geringen Affinität für Wasser in solche mit einer grösseren Wasseraffinität umgewandelt werden. Wachstum in der lebenden Materie ist dadurch charaliterisiert, dass es "von innen nach aussen" zustande kommt, eine Bedingung, die nicht in dem Wachstum oder der Ersetzung von Kristallen, jedoch in der Quellung irgendeiner der sogenannten hydrophilen (Perrin) Kolloide erfüllt wird. 
Im folgenden Argument wird es sich herausstellen; dass jede dieser Aufstellungen ohne Modifikation für die Wasserabsorption durch Froschmuskeln von Gültigkeit ist.

\section{Versuchsmethoden.}

Zwei Arten Froschmuskeln wurden in diesen Versuchen benutzt. Für die meisten Experimente diente der gewöhnliche Laubfrosch (Hyla), der besonders zugänglich war. Wegen der Kleinheit der Frösche wurden gewöhnlich beide von der Haut befreiten Hinterbeine benutzt. Der Kontrolle wegen wurden zuweilen die Beine vermittelst eines Schnittes durch das Becken zerteilt und in verschiedene Lösungen gebracht. Bei den Versuchen, in welchen nur der Gastrocnemius benutzt wurde, wobei grosse, essbare Frösche als Ausgangsmaterial dienten, assistierte mir Dr. Gertrude Moore, was ich mit grossem -Vergnügen hier anerkenne.

Die Lösungen (je $110 \mathrm{cem}$ ), in welche die frisch präparierten Muskeln eingetaucht wurden, wurden in leicht bedeckte Glasschalen gegossen. Die Muskeln wurden auf Filtrierpapier von gleichem Gewicht gewogen. Nach Aushebung aus einer der Lösungen zwecks des Wägens wurden die Muskeln auf ein Stüek Fliesspapier gelegt und mit einem zweiten Stück schnell abgetrocknet. Gleich nach der Gewichtsbestimmung, die nie mehr als 3 Minuten verlangte, und während welcher Zeit die Muskeln keine bemerkenswerte Gewichtsänderung erfuhren, wurden die Muskeln in ihre respektiven Lösungen zurückgebracht. Der dureh ungleiches Trocknen der Muskeln verursachte Gewichtsfehler liegt im dritten Dezimalpunkt. In vielen der Lösungen quellen die Muskeln so stark auf, dass Teilchen derselben verloren gehen. Nicht allein Iösen sich die Muskeln auf; sondern äussere Teilchen der gelatinösen Masse kleben am Filtrierpapier usw. fest. Hierdurch wird ein beträchtlicher Versuchsfehler in die späteren Gewichtsbestimmungen gebracht.

Ich war bestrebt, die Muskeln der verschiedenen Frösche so weit wie möglich im selben Zustand zu bekommen. Die Frösche wurden frisch gefangen gebraucht und während des Aufenthaltes im Laboratorium im selben Glasgefäss, das ein wenig Laub und genügend Wasser enthielt, : um die umgebende Luft feucht zu erhalten, aufbewahrt. Nichtsdestoweniger zeigten die Muskeln ansehnliche individuelle Verschiedenheiten, wenn sie in dieselben Lösungen gebracht wurden. Deshalb und um eine bessere Vorstellung über den Prozess 
der Wasserabsorption und Sekretion durch Froschmuskeln zu erhalten, habe ich Durchschnittsbestimmungen und Mittelwerte vermieden. Sonst wäre es auch unmöglich gewesen die Absorptionskurven, die einen besseren Überblick der sich in den Muskeln abspielenden Änderungen gestatten, als Zahlenreihen, die durch Wägungen ad libitum gewonnen sind, graphisch darzustellen. Die Versuche sind so zeitraubend, dass es unmöglich ist, so grosse Versuchsreihen über jeden einzelnen Punkt anzuführen, ais ich wohl wünschen möchte; nichtsdestoweniger sind die experimentellen Ergebnisse so auffallend, dass sie die gezogenen Schlussfolgerungen völlig rechtfertigen.

\section{Versuche.}

I.

Der grösste Wasserbetrag, der durch einen Frosehmuskel bei irgendeinem meiner Versuche jemals absorbiert wurde, beträgt weniger als $21 / 2 \mathrm{mal}(246,66 \%)$ des Anfangsgewichtes des Muskels. Da der Muskel ungefähr $75 \%$ Wasser und nur $1 \%$ Asche enthält, so können wir annehmen, dass zirka ein Viertel des Gewichtes des gewöhnlichen Muskels aus verschiedenen organischen Substanzen besteht. Beinahe alle diese gehören in die Gruppe der Kolloide. Hiernach berechnet, würde $1 \mathrm{~g}$ getrockneter Muskel $4 \mathrm{~g}$ feuchter, normaler Muskelsubstanz entsprechen, welche die Fähigkeit hat, genügend Wasser zu absorbieren (250\%), um $14 \mathrm{~g}$ zu wiegen. Nach dem Trockengewicht berechnet, bedeutet dies also, dass die Muskelkolloide unter gewissen Bedingungen die Fähigkeit haben, das 13 fache $(1300 \%)$ ihres Gewichtes Wasser zu absorbieren. Damit soll jedoch nicht gesagt werden, dass dies die Maximum-Absorptionsziffer irgendeines Muskels ist. Es registriert nur die höchste Wasserabsorption, die in irgendeinem der im folgenden beschriebenen Versuche gefunden wurde. Gewöhnlich können wir nur mit einer Verdoppelung des Gewichtes des normalen Muskels rechnen, oder im äussersten Fall auf eine Zunahme von $150 \%$. Dies aufs Trockengewicht des Muskels berechnet, würde einer Zunahme von nur 700 bis $900 \%$ entsprechen.

Gewöhnliches, getrocknetes Fibrin hat die Fähigkeit, Wassermengen zu absorbieren, die die grössten Mengen, welche durch Froschmuskeln bei meinen Versuchen jemals absorbiert wurden, weit übertreffen. Hierdurch wird bewiesen, dass ein einfaches Kolloid 
die Fähigkeit besitzen kann, genügend Wasser zu absorbieren, um imstande zu sein, für all das jemals durch einen Muskel absorbierte Wasser. Erklärung zu geben. 1 g getrocknetes Fibrin absorbiert mit Leichtigkeit 10-20 mal das Eigengewicht an Wasser in einer Salzsäurelösung von bestimmter Konzentration. Keineswegs bedeuten diese Zahlen die grössten Mengen Wasser, die von Fibrin absorbiert werden können. In Kalilauge von bestimmter Konzentration kann getrocknetes Fibrin 30, zuzeiten sogar beinahe 40 mal das Eigengewicht an Wasser absorbieren (3000-4000\%).

Es ist $z u$ erwähnen, dass der Hauptbestandteil der sich im Muskel befindenden organischen Materie aus Myosinogen und Myosin besteht. Die chemischen und physikalischen Ähnlichkeiten, die zwischen diesen Körpern und Fibrinogen und Fibrin existieren, sind zu wohl bekannt, um besonders hervorgehoben zu werden; und es liegt der Gedanke nahe, dass die Analogie der Quellung des Fibrins und der Quellung des Froschmuskels am Ende durch eine Analogie der Quellung des Fibrins und des Myosins bestimmt ist. Jedoch glaube ich nicht, dass dies Kolloid allein dabei eine Rolle spielt. Bei Versuchen, in welchen die Muskeln bedeutend Wasser absorbieren, bemerkt man, dass die die Gelenke umgebenden Bindegewebe, Sehnen und Knorpel in klare, homogene, gelatinöse Massen aufquellen.

\section{II.}

Froschmuskel quillt mehr in der Lösung irgendeiner Säure als in reinem Wasser, aber die Quellung ist bei verschiedenen Säuren verschieden stark. Diese Tatsachen treten in den Fig. $1,4,7$ und 10 zutage. Da all die Kurven in dieser Abhandlung nach derselben Skala gezeichnet sind, können sie direkt miteinander verglichen werden: Fig. 1, 4, 7 und 10 beziehen sich auf die Quellung von Laubfroschhinterbeinen, nachdem dieselben in $110 \mathrm{ccm}$ destillierten Wassers oder $100 \mathrm{ccm}$ destillierten Wassers plus $10 \mathrm{ccm} 1 / 10$ norm. Säure eingebracht wurdeu. Fig. 4, 7 und 10 zeigen klar, dass die höchste Quellungskurve in reinem Wasser bei weitem nicht an die in Salzsäurelösung heranreicht. Diese sowohl als alle dieser Mitteilung beigelegten Kurvenzeichnungen sind durch Aufzeichnung der Prozente Gewichtszunahme oder Abnahme, welche die Muskeln in ihren respektiven Lösungen direkt nach Entfernung von den frischgetöteten Fröschen erleiden, 
gewonnen. Während die HCl-Kurve steil emporsteigt, um langsamer wieder zu fallen, ist die in reinem Wasser gewonnene Kurve komplizierter. Hier haben wir ein rasches Steigen, dann ein Abnehmen, und wieder mal ein Ansteigen, das beibehalten wird. Diese Gewichtsabnahme des Muskels nach der anfänglichen Zunahme ist für die Muskelquellung in destilliertem Wasser sehr charakteristisch. Die Gewichtsbestimmungen und Berechnungen, aus welchen die Kurven 4; 7 und 10 konstruiert sind, sind in den Tabellen IV, VII und $X$ wiedergegeben.

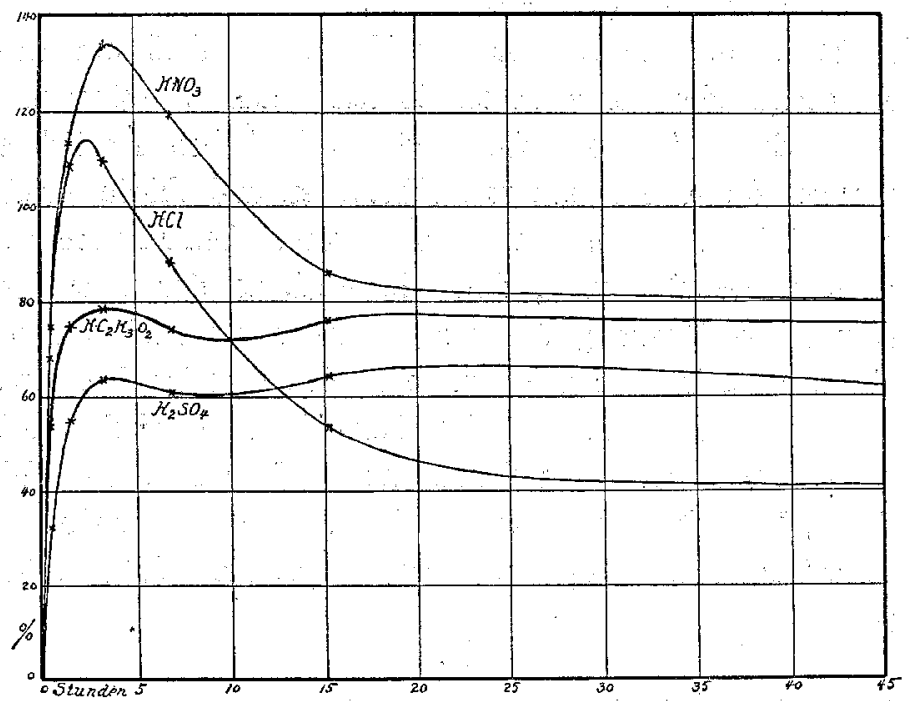

Fig. 1.

Wie viel mehr Froschmuskel in einigen Säuren quillt als in anderen, lässt sich aus Fig. 1 klar ersehen. Die Säuren waren alle $1 / 110$ normal, bereitet durch Verdünnung von $10 \mathrm{ecm}$ der entsprechenden $1 / 10$ norm. Säure mit 100 cem destillierten Wassers. Die vier benutzten Säuren folgen nach der Reihe:

Salpetersäure, Salzsäure, Essigsäure, Schwefelsäure.

Die Salzsäure hätte leicht das erste Glied dieser Reihe bilden können. Tatsächlich steht sie gewöhnlich vor der Salpetersäure, wie deutlich hervortritt, wenn die niedrige in Fig. 1 wiedergegebene HCl-Kurve mit den HCl-Kurven der Fig. 4, 6, 9, 11 und 13 verglichen wird. Ohne darauf zu bestehen - bis weitere Versuche angestellt sind -, dass die Reihenfolge, nach welcher Säuren die Quellung von Froschmuskeln begünstigen, identisch ist mit der, nach 
welcher diesetben Säuren Fibrin zum Quellen bringen, haben die zwei Reihen doch viel gemeinsames. Bei unseren Versuchen mit Fibrin fanden wir, dass diese Substanz in Salzsäure stärker quillt als in einer äquinormalen Salpetersäure und in dieser wiederum stärker als in einer äquinormalen Essigsäure. Das letzte Glied der Reihe bildet Schwefelsäure. Schwefelsäure steht nur wenig höher als reines Wasser in den Quellungsversuchen über Fibrin, und es übertrifft destilliertes Wasser auch nur in geringem Maasse bei Versuchen über die Quellung von Muskeln. Es ist bemerkenswert, dass die Muskeln in Schwefelsäure sowie in Essigsäure eine Zeitlang nach der ersten grossen Gewichtszunahme an Gewicht abnehmen, um dann zum zweitenmal zu schwellen. Solch ein temporärer Gewichtsverlust kommt auch bei Muskeln vor, die in reinem Wasser behandelt wurden.

Fig. 1 ist nach den in Tabelle I mitgeteilten Versuchen konstruiert. Die erste Zahl in jeder Zahlenreihe aller dieser Tabellen gibt das ursprüngliche Gewicht der Muskeln an. Nach jeder der Gewichtsbestimmungen steht in Klammern das Prozent der Gewichtszunahme oder -abnahme. Eine Gewichtszunahme kennzeichnet sich durch ein $(+)$, eine Gewichtsabnahme durch (-).

\section{Tabelle I.}

Laubfroschmuskeln. Beide Hinterbeine wurden in jeder der Lösungen benutzt.

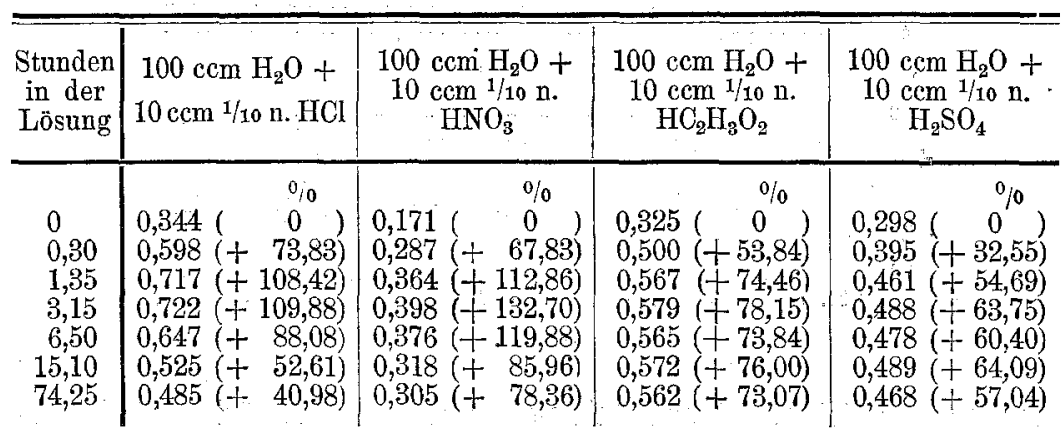

III.

Das Hinzufügen irgendeines Salzes zu der Säurelösung verringert die Quellung der Muskel in jener Lösung. Ein Blick auf Fig. 2, 3 und 4 beweist dies besser als viele Worte. Fig. 2 und 3 sind aus Beobachtungen, die sich auf die Gastrocnemien von grossen essbaren Fröschen beziehen, kon- 
struiert. Fig. 4 ist Versuchen, in welchen die beiden Hinterbeine von Laubfröschen benutzt wurden, entnommen. Ich bemerke hierzu, dass die Quellung in reinen Säurelösungen für den Gastrocnemius allein durchschnittlich etwas höher ist als in Laubfroschbeinen. Der Ursprung dieser Erscheinung ist wohl nicht in wirklichen Untersehieden der zwei Arten Muskulatur zu suchen, sondern darin, dass Knochen in den Laubfroschhinterbeinen vorhanden sind, die in

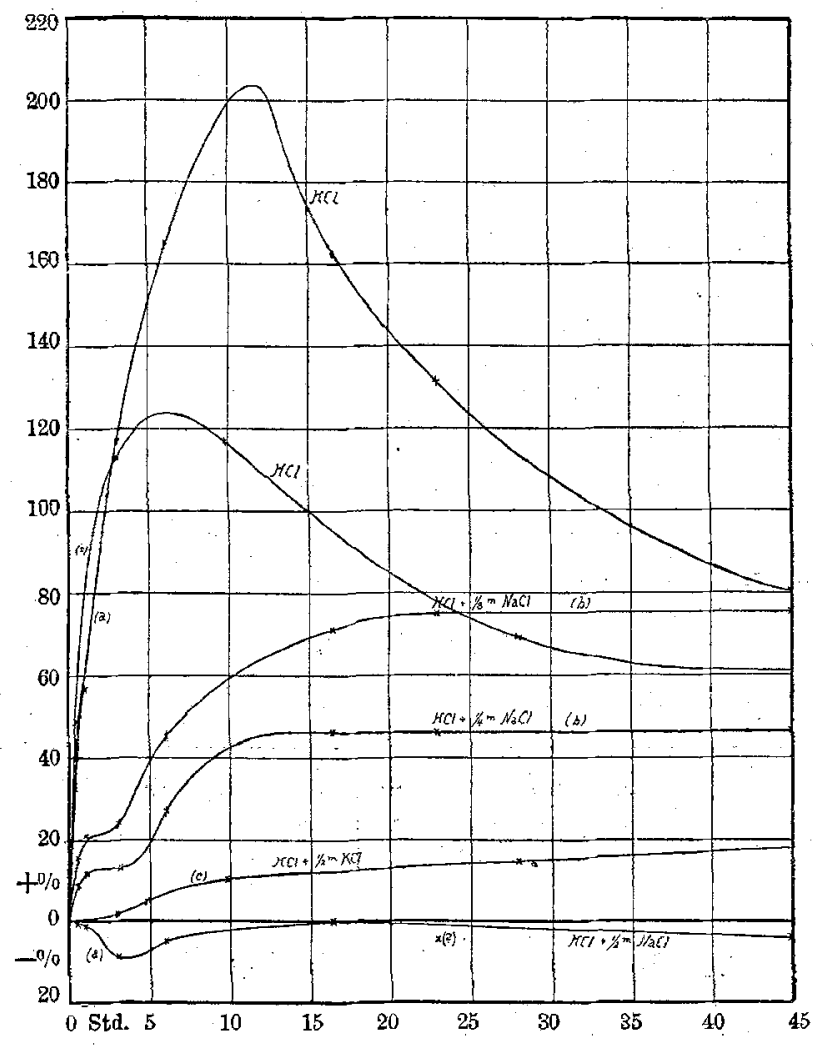

Fig. 2.

manchen der Lösungen sich teilweise auflösen und lösliche Calcium(und andere) Salze bilden. Wie später gezeigt werden soll, vermindern Calciumsalze sehr stark die Quellung vón Froschmuskeln in Säurelösungen.

Fig. 2 und 3 zeigen auch, dass $\mathrm{Muskel}$ desto weniger in einer Säurelösung quillt, je höher die Konzentration eines Salzes in der Lösung ist. In Figur 2 sind Kurven für drei versehiedene Kochsalzkonzentrationen aufgezeichnet. Die 
Lösungen wurden durch Zufügung von $10 \mathrm{ccm}{ }^{1 / 10}$ norm. Salzsäure zu je $100 \mathrm{ccm} 1 / 8$ molek., 1/4 molek. und 1/2 molek. Kochsalzlösung bereitet. Der Unterschied zwischen diesen Kurven und den durch $\mathrm{Zu}$ fügung von $10 \mathrm{cem}{ }^{1 / 10}$ norm. Salzsäure zu reinen Wasser gewonnenen

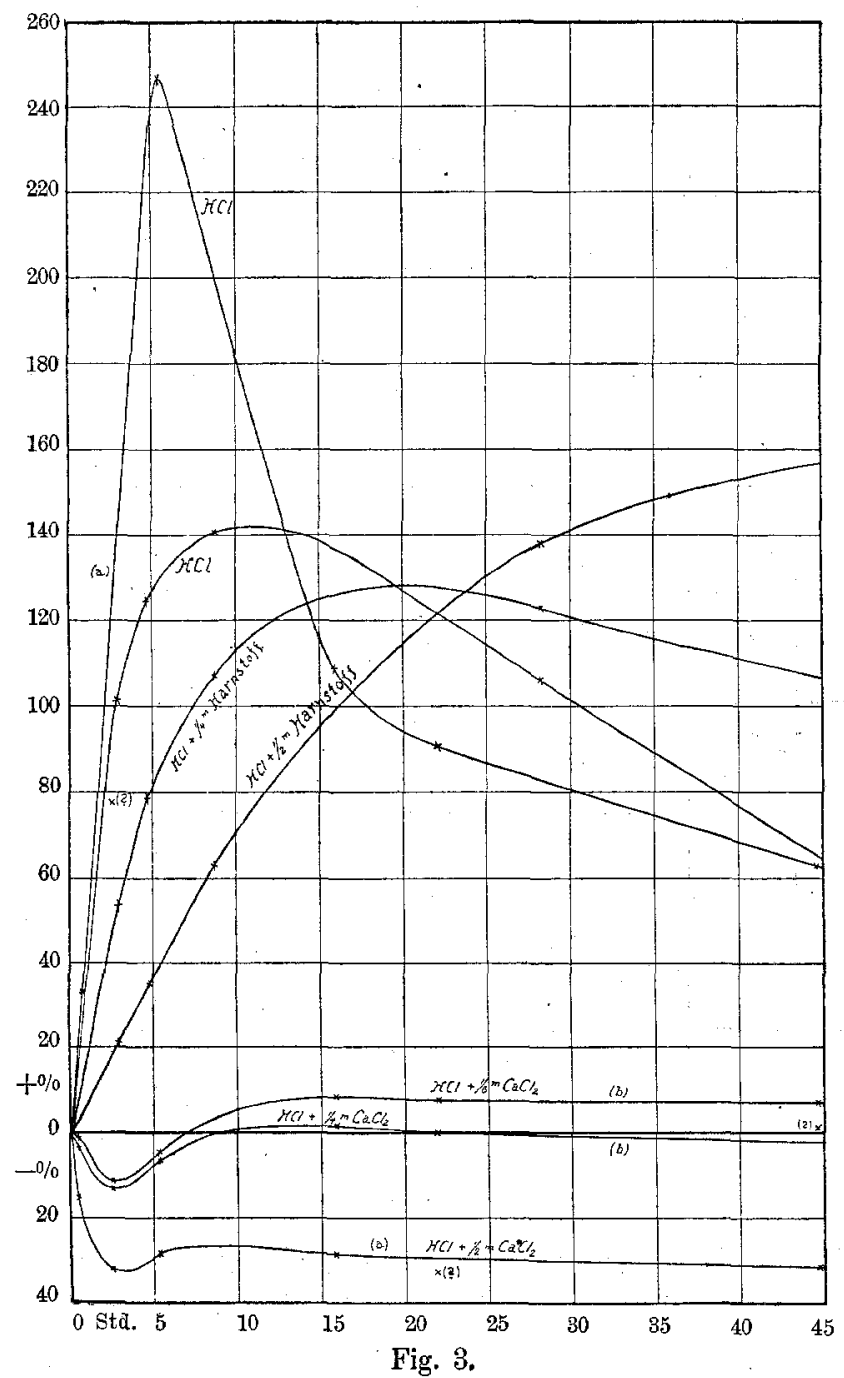

ist sehr auffallend. Zwei solche reine Säurekurven sind in Fig. 2 aufgezeichnet; $(a)(a),(b)(b)$ und $(c)(c)$ bedeuteñ je die zwei Gastrocnemien eines vom selben Froseh herstammenden Paars Muskeln. -

Man ersieht aus der Zeichnung, dass durch Gebrauch einer genügend hohen Kochsalzkonzentration die Quellung hervorrufende Fähig- 
keit der Salzsäure ganz unterdrückt werden kann. Die nach Gebrauch einer ${ }^{1}{ }^{\prime}$ ' molek. Kochsalzlösung gewonnene Kurve liegt ganz unterhalb der Nullinie. Äquimolekulare Lösungen anderer Salze mögen stärker oder schwächer als Kochsalz wirken (siebe unten). Dieser Antagonismus zwischen Säuren und Salzen ist bis jetzt nur wenig erforscht worden, obschon er nicht wenig zur Erkläruung einer ganzen

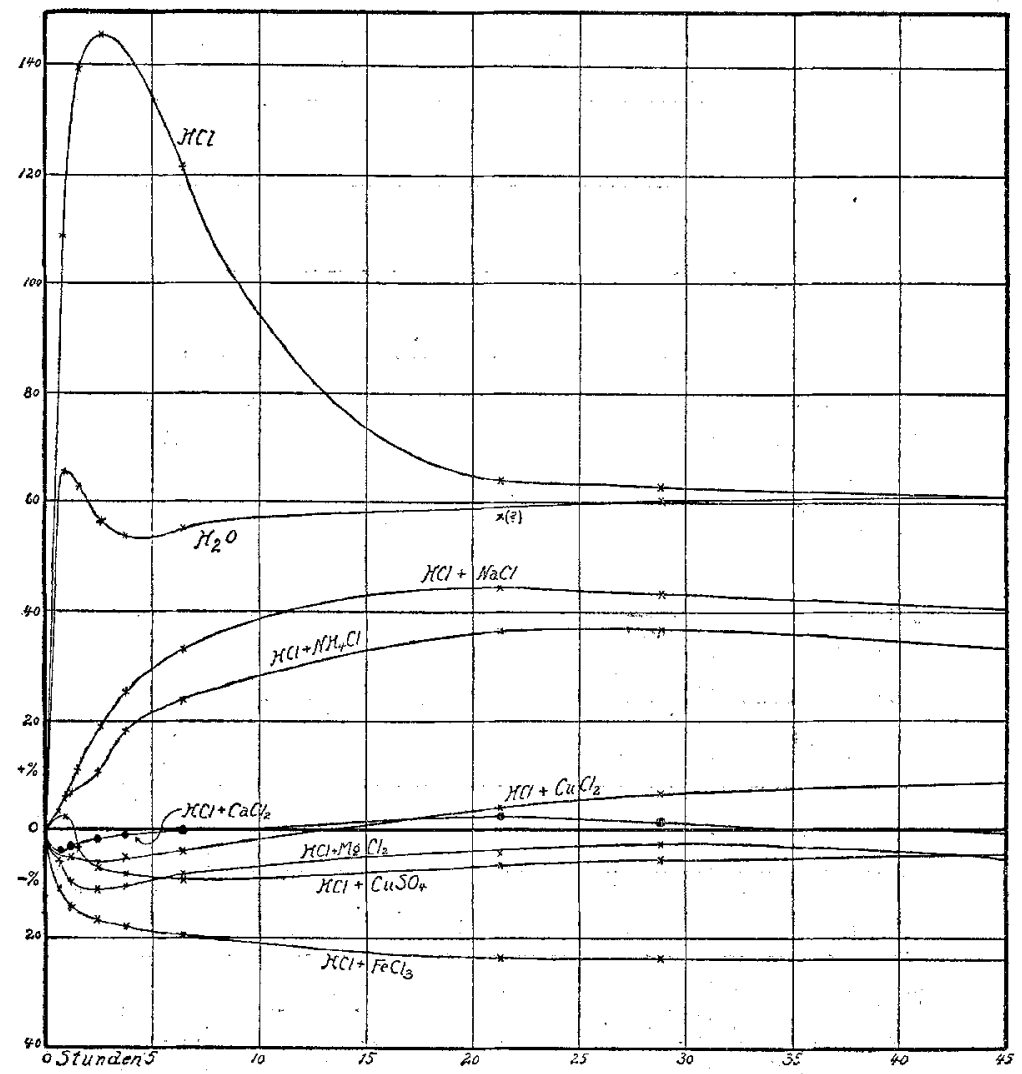

Fig. 4.

Reihe physiologischer und pathologischer Probleme, in welchen Säuren eine Rolle spielen, beizutragen verspricht. Vorläufig wird es genügen, darauf aufmerksam zu machen, dass, dieser Antagonismus wahrscheinlich für die Immunität von Tieren gegen selbst enorme Dosen Säuren [und Alkalien ${ }^{1}$ )] wenigstens zum Teil verantwortlich ist; und dies ganz abgesehen von der Tatsache, dass gewisse Gewebesalze die Fähig-

1) Nach Versuchen, die bald veröffentlicht werden sollen, besteht ein Antagonismus sowohl zwischen Alkalien und Salzen wie zwischen Säuren und Salzen. 
heit besitzen, starke Säuren (oder Basen) zu nentralisieren. Wie diese Versuche mit Muskel und unsere früheren über die Quellung des Fibrins dartun, besteht ein Antagonismus zwischen neutralen Salzen und Säuren. Beweise für die Existenz eines solchen Antagonismus sind in Änderungen in Kolloid oder Kolloide zu finden, und diese bilden, wie wir wissen, die physikalische Basis all jener Erscheinungen, die wir als "lebend" charakterisieren.

Die quellungsherabsetzende Wirkung eines Salzes auf einen in Säure sich befindenden Muskel ist nicht auf eine einfache Herabsetzung der elektrolytischen Dissoziation der Säure zurückzuführen. Nicht allein ist der Grad einer solchen Herabsetzung zu klein, um die enorme Abnahme des Quellungsgrads zu erklären, sondern eine solche Erklärung würde auch nur dann stichhaltig sein, wenn das Salz ein mit der Säure gemeinsames Ion enthält. Weiter sollten Salze, die den Dissoziationsgrad gleich verringern, den Quellungsgrad gleich herabsetzen, was sie nicht tun.

In Fig. 3 sind Kurven wiedergegeben, die die Gewichtsveränderungen veranschaulichen, welche Gastrocnemiusmuskeln in reinen Salzsäurelösungen und in gleichkonzentrierten Salzsäurelösungen, denèn verschiedene Mengen Chlorcalcium zugesetzt sind, erleiden. Die Lösungen wurden durch Zufügung von $10 \mathrm{ccm}$ $1 / 10$ norm. Salzsäure zu je $100 \mathrm{ccm}$ destillierten Wassers, oder $1 / 2$ molek., $1 / 4$ molek. und $1 / 8$ molek. Chlorcalciumlösung bereitet. Die bohe reine HCl-Kurve (die höchste, die ich in irgendeinem meiner Versuche gewonnen habe) steht der durch Zufügung von 1/2 molek. Chlorcalcium gewonnenen stark entgegen. Die Kurven $(a)(a)$ und (b) (b) beziehen sich je auf die zwei Gastrocnemien eines vom selben Frosch herstammenden Paars Muskeln.

Wenn Fig. 2 mit Fig. 3 verglichen wird, so tritt der grosse Untersehied in den quellungsherabsetzenden Fähigkeiten äquimolekularer Chlornatrium- und Chlorealciumlösungen klar zutage. Auf diesen Punkt wird später wieder zurückgekommen. In Fig. 2 ist zum Vergleich eine durch Zufügung von $10 \mathrm{ccm} \mathrm{1/10} \mathrm{norm} \mathrm{Salzsäure} \mathrm{zu}$ 100 ccm einer ${ }^{1 / 2}$ molek. Chlorka li u m lösung gewonnene Kurve aufgezeichnet. Es ist aus diesen Zeichnungen leicht zu ersehen, dass Chlorkalium die Quellung vonFroschmuskeln weniger herabsetzt als eine gleiche Konzentration Chlornatrium, und dass dieses wiederum in der Hinsicht weniger fähig ist als äquimolekulares Chlorcalcium. 
Die Versuchsprotokolle, aus denen die in Fig. 2 und 3 sich befindenden Kurven konstruiert wurden, sind in den Tabellen II und III wiedergegeben.

Tabelle II.

Gastrocnemiusmuskeln. Glieder desselben Muskelpaares sind durch denselben Buchstaben angedeutet.

\begin{tabular}{|c|c|c|c|c|}
\hline $\begin{array}{l}\text { Stunden } \\
\text { in der } \\
\text { Lösung }\end{array}$ & $\begin{array}{l}100 \mathrm{ccm} \mathrm{H}_{2} \mathrm{O}+ \\
10 \mathrm{~cm}^{1 / 10 ~ n . ~ H C l}\end{array}$ & $\begin{array}{l}100 \mathrm{ccm} 1 / 2 \mathrm{~m} . \\
\mathrm{NaCl}+10 \mathrm{ccm} \\
1 / 10 \mathrm{n} . \mathrm{HCl}\end{array}$ & $\begin{array}{c}100 \mathrm{ccm} 1 / 4 \mathrm{~m} . \\
\mathrm{NaCl}+10 \mathrm{ccm} \\
1 / 10 \mathrm{n} . \mathrm{HCl}\end{array}$ & $\begin{array}{c}100 \mathrm{ccm} 1 / 8 \mathrm{~m} . \\
\mathrm{NaCl}+10 \mathrm{ccm} \\
1 / 10 \mathrm{n} . \mathrm{HCl}\end{array}$ \\
\hline $\begin{array}{r}0 \\
0,25 \\
1,00 \\
2,55 \\
5,55 \\
16,25 \\
22,50 \\
45,00 \\
70,25 \\
95,00\end{array}$ & $\begin{array}{c} \\
0,81\left(\begin{array}{c}0 \\
0\end{array}\right) \\
1,20(+48,14) \\
1,27(+56,79) \\
1,75(+116,05) \\
2,14(+165,43) \\
2,12(+161,72) \\
1,87(+130,86) \\
1,46(+80,24) \\
1,28(+58,02) \\
1,11 \text { (+ } 37,03) \\
\text { (a) }\end{array}$ & $\begin{array}{c}0,85 \\
0,85\left(0^{0}\right) \\
0,84(-1,17) \\
0,84(-1,17) \\
0,77(-9,41) \\
0,80(-5,88) \\
0,85(0) \\
0,81(-4,70) \\
0,82(-3,53) \\
0,80(-5,88) \\
0,81(-4,70) \\
\text { (a) }\end{array}$ & $\begin{array}{c}{ }^{0 / 0} \\
0,63\left({ }^{0}\right) \\
0,68(+7,98) \\
0,70(+11,11) \\
0,71(+12,69) \\
0,80(+26,98) \\
0,92(+46,03) \\
0,92(+46,03) \\
0,92(+46,03) \\
0,92(+46,03) \\
0,92(+46,03) \\
\text { (b) }\end{array}$ & 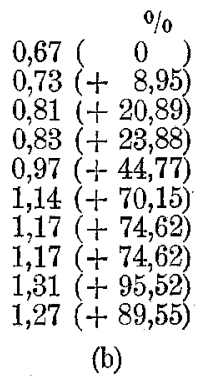 \\
\hline & $\begin{array}{l}\text { Stunden in } \\
\text { der Lösung }\end{array}$ & $\begin{array}{c}100 \mathrm{ccm} \mathrm{H}_{2} \mathrm{O}+ \\
10 \mathrm{ccm} 1 / 10 \mathrm{H} . \mathrm{HCl}\end{array}$ & \multicolumn{2}{|c|}{$\begin{array}{c}100 \mathrm{ccm}^{1 / 2 \mathrm{~m}} . \mathrm{KCl}+ \\
10 \mathrm{ccm} 1 / 10 \mathrm{n} . \mathrm{HCl}\end{array}$} \\
\hline & $\begin{array}{r}0 \\
2,50 \\
4,50 \\
9,50 \\
27,55 \\
52,20 \\
77,35\end{array}$ & $\begin{array}{c} \\
1,27\left(\begin{array}{c}0 \\
0\end{array}\right) \\
2,72(+114,49) \\
2,82(+122,04) \\
2,75(+116,53) \\
2,15(+69,29) \\
1,20(-5,51) \\
1,00(-21,26) \\
\text { (c) }\end{array}$ & \multicolumn{2}{|c|}{ 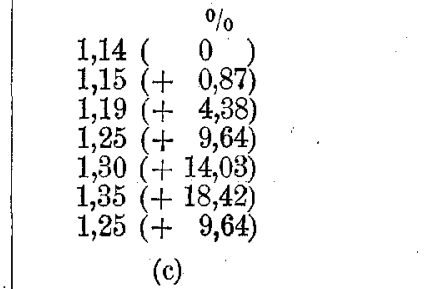 } \\
\hline
\end{tabular}

Tabelle III.

Gastrocnemiusmuskeln. Glieder desselben Muskelpaares sind durch denselben Buchstaben angedeutet.

\begin{tabular}{|c|c|c|c|c|}
\hline $\begin{array}{c}\text { Stunden } \\
\text { in der } \\
\text { Lösung }\end{array}$ & $\begin{array}{l}100 \mathrm{ccm} \mathrm{H}_{2} \mathrm{O}+ \\
10 \mathrm{ccm}^{1} / 10 \mathrm{n} . \mathrm{HCl}\end{array}$ & $\begin{array}{c}100 \mathrm{ccm} 1 / 2 \mathrm{~m} \\
\mathrm{CaCl}_{2}+ \\
10 \mathrm{ccm}^{1} / 10 \mathrm{n} . \mathrm{HCl}\end{array}$ & 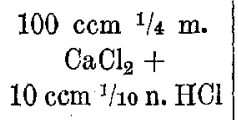 & $\left\{\begin{array}{c}100 \mathrm{ccm}^{1 / 8 \mathrm{~m}} \\
\mathrm{CaCl}_{2}+ \\
10 \mathrm{ccm}^{1 / 10 \mathrm{n} . \mathrm{HCl}}\end{array}\right.$ \\
\hline $\begin{array}{r}0 \\
0,25 \\
2,25 \\
5,20 \\
15,45 \\
21,55 \\
44,40\end{array}$ & $\begin{array}{c}0 / 0 \\
0,75\left(\begin{array}{c}0 \\
0\end{array}\right) \\
1,00(+33,33) \\
1,33(+77,33) \\
2,60(+246,66) \\
1,57(+109,33) \\
1,43(+90,66) \\
1,22(+62,66) \\
1,11 \text { (+ } 48,00) \\
\text { (a) }\end{array}$ & $\begin{array}{l}0 / 0 \\
0,77\left(\begin{array}{c}0 \\
0,75\end{array}\right) \\
0,65(-15,58) \\
0,52(-32,47) \\
0,55(-28,57) \\
0,55(-28,57) \\
0,52(-32,47) \\
0,53(-31,16) \\
0,53(-31,16) \\
(a)\end{array}$ & 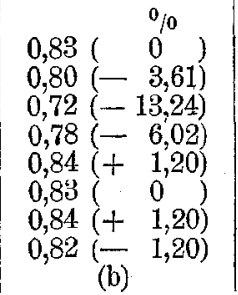 & $\begin{array}{c}0 \% \\
0,85\left(\begin{array}{c}0 \\
0\end{array}\right) \\
0,84(-1,17) \\
0,74(-12,94) \\
0,81(-4,70) \\
0,92(+8,28) \\
0,91(+7,05) \\
0,91(+7,05) \\
0,90(+5,88) \\
\text { (b) }\end{array}$ \\
\hline
\end{tabular}


IV.

Äquimolekulare Lösungen verschiedener Salze bringen nicht eine gleiche Quellungsverringerung des Froschmuskels hervor. Auf diese Tatsache ist schon in den vorhergehenden Paragraphen aufmerksam gemacht worden, als gezeigt wurde, dass eine Chlorcalciumlösung die Quellung eines Muskels in einer Säurelösung mehr hemmt, als eine äquimolekulare Chlornatriumlösung, und diese mehr als eine gleichmolekulare Chlorkaliumlösung. Gertrude Moore und ich fanden die gleiche Anordnung der Kationen ( $\mathrm{K}, \mathrm{Na}, \mathrm{Ca}$ ) bei unseren Versuchen über die quellungshemmende Wirkung von Salzen auf die Quellung von Fibrin in Säurelösungen.

In Fig. 4 sind die Resultate einer etwas längeren Versuchsreihe mit den Chloriden verschiedener Metalle aufgezeichnet. Laubfroschhinterbeine wurden bei den Versuchen benutzt. Es wurden immer je $10 \mathrm{ccm}$ einer $1 / 10$ norm. Salzsäure $100 \mathrm{ccm}$ der entsprechenden $1 / 4$ molek. Salzsäure zugesetzt Die in reinem Wasser und in reiner Salzsäurelösung gewonnenen Kurven sind zum Zweek des Vergleichs und zur Kontrolle eingeschaltet. Es ist klar, das die Chloride der verschiedenen Metalle eine bestimmte Reihenfolge haben. Während einige einfach die Quellung verringern, bringen andere einen wirklichen Gewichtsverlust in den Muskeln zustande, selbst bei Gegenwart der Salzsäure. Die Kationen ordnen sich ungefähr nach folgender Reihe:

$$
\mathrm{Na}^{+}, \mathrm{NH}_{4}^{+}, \mathrm{Ca}^{++}, \mathrm{Cu}^{++} \text {(ic) (?), } \mathrm{Mg}^{++}, \mathrm{Fe}^{+++} \text {(ic). }
$$

Es steht ein Fragezeichen hinter Kupfer, da dessen Kurve in den ersten Versuchsstunden unter. der Calciumkurve steht, nachher sie aber übersteigt. Die Kurve für schwefelsaures Kupfer ist zu der Beobachtung eingeschaltet, dass mit einem gemeinsamen Kation ein Sulphat unter einem Chlorid steht $\left(\mathrm{Cu} \mathrm{SO}_{4}\right.$-Kurve unter der $\mathrm{Cu} \mathrm{Cl}_{2}$ Kurve). Die in Fig. 4 aufgezeichneten Kurven sind aus den in Tabelle IV (S. 82) wiedergegebenen Beobachtungen konstruiert.

In Fig. 5 und 6 sind die Wirkungen einer Reihe Acetate auf die Quellung von Laubfroschmuskeln in Salzsäure wiedergegeben. Fig. 5 bezieht sich auf Versuche, bei welchen $10 \mathrm{ccm}$ einer 1/10 norm. Salzsäure je $100 \mathrm{ccm}$ einer $1 / 4$ molek. Kaliumacetat-, Natriumacetat- und Calciumacetatlösung zugesetzt wurden; Fig 6 auf solche, bei welchen dasselbe Säurequantum $100 \mathrm{ccm} 1 / 2$ molek. 
Tabelle IV.

Laubfroschmuskeln. Beide Hinterbeine wurden in jeder der Lösungen benutzt.

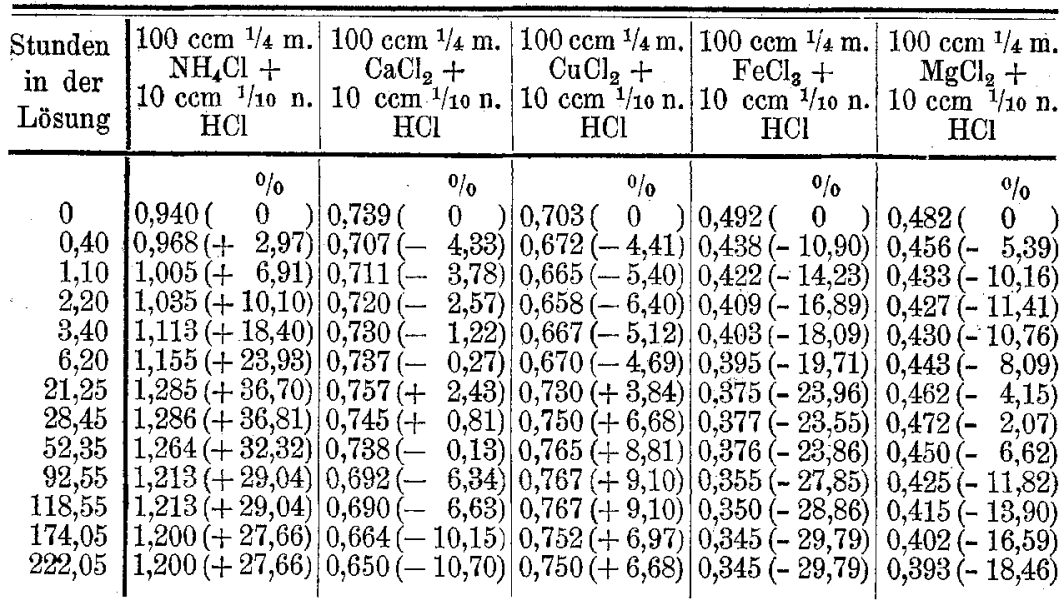

\begin{tabular}{|c|c|c|c|c|}
\hline $\begin{array}{l}\text { Stunden } \\
\text { in der } \\
\text { Lösung }\end{array}$ & $\begin{array}{c}100 \mathrm{ccm} 1 / 4 \mathrm{~m} . \\
\mathrm{NaCl}+ \\
10 \mathrm{ccm} 1 / 10 \mathrm{n} . \\
\mathrm{HCl}\end{array}$ & $\begin{array}{c}100 \mathrm{ccm}^{1 / 4} \mathrm{~m} . \\
\mathrm{CuSO}_{4}^{+} \\
10 \mathrm{ecm}^{1 / 10} \mathrm{n} . \\
\mathrm{HCl}\end{array}$ & $\begin{array}{c}100 \mathrm{cem} \mathrm{H}_{2} \mathrm{O}+ \\
10 \mathrm{ccm} 1 / 10 \mathrm{n} . \\
\mathrm{HCl}\end{array}$ & $110 \mathrm{ccm} \mathrm{H}_{2} \mathrm{O}$ \\
\hline $\begin{array}{r}0 \\
0,50 \\
1,30 \\
2,30 \\
3,40 \\
6,20 \\
21,35 \\
28,55 \\
52,55 \\
93,25 \\
119,10 \\
174,10 \\
222,10\end{array}$ & 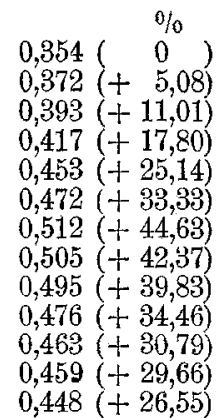 & $\begin{array}{l}0 / 0 \\
0,312(0) \\
0,320(+2,56) \\
0,301(-3,52) \\
0,291(-6,39) \\
0,283(-9,29) \\
0,283(-9,29) \\
0,292(-6,41) \\
0,295(-5,45) \\
0,297(-4,80) \\
0,298(-4,49) \\
0,297(-4,80) \\
0,297(-4,80) \\
0,300(-3,84)\end{array}$ & $\begin{array}{c}\text { \% } \\
0,269\left(\begin{array}{c}0 \\
0,\end{array}\right) \\
0,562(+108,88) \\
0,645(+139,77) \\
0,660(+145,35) \\
0,648(+140,89) \\
0,543(+101,85) \\
0,440(+63,56) \\
0,439(+63,19) \\
0,431(+60,22) \\
0,397(+47,58) \\
0,315(+17,32) \\
0,245(-\quad 8,88) \\
0,250(-\quad 7,06)\end{array}$ & 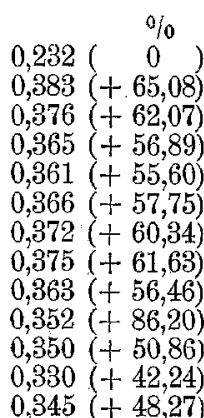 \\
\hline
\end{tabular}

Lösungen derselben Salze zugefügt wurde. In jede der beiden Zeichnungen ist eine reine Salzsäurekurve zum Vergleich eingeschaltet. Für eine Reihe Acetate sehen wir hier wieder die Anordnung der Kationen:

$$
\mathrm{K}^{+}, \mathrm{Na}^{+}, \mathrm{Ca}^{+}+
$$

Bei den späteren Versucbsstunden in Fig. 6 liegt die Kaliumkurve unterhalh deren für Natrium und Calcium. Solche Verhaltungsänderung in den verschiedenen Muskeln ist schwer zu erklären.

Die in diesen Paragraphen beschriebenen Versuche ermöglichen es uns, die auf Seite 89 angegebene Kationenliste etwas zu verlängern. Ohne Zweifel steht Kalium vor Natrium. Wird die in 
Über die Analogie zwischen der Wasserabsorption durch Fibrin etc. 83 Fig. 9 gezeichnete Baryumnitratkurve mit der in Fig. 10 wiedergegebenen Calciumnitratkurve verglichen, so kann ein ungefährer

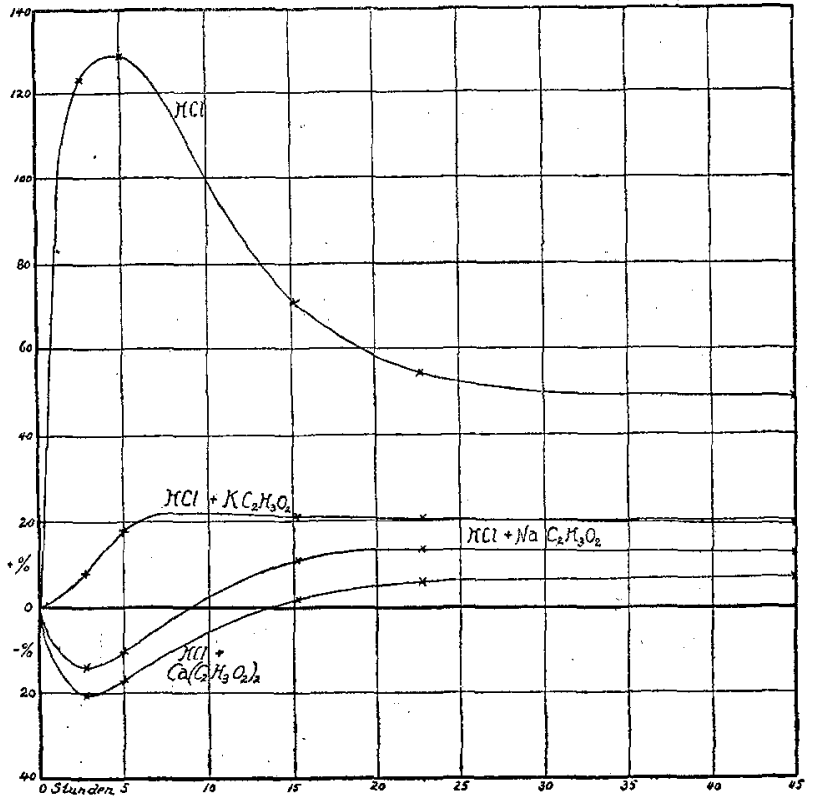

Fig. 5.

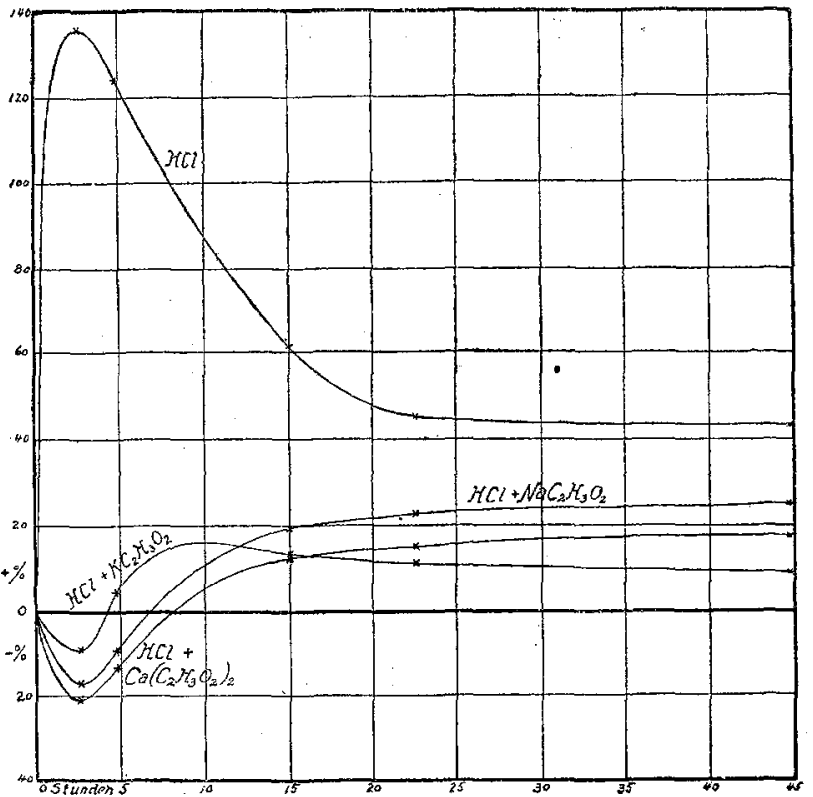

Fig. 6. 
Platz für Baryum gefunden werden, der offenbar nahe Calcium in unserer Kationenreihe zu suchen ist.

Die in Fig. 5 und 6 sich befindenden Kurven sind den in Tabelle V und VI wiedergegebenen Versucbszahlen entnommen.

\section{Tabelle V.}

Laubfroschmuskeln. Beide Hinterbeine wurden bei jedem der Versuche benutzt.

\begin{tabular}{|c|c|c|c|c|}
\hline $\begin{array}{l}\text { Stunden } \\
\text { in der } \\
\text { Lösung }\end{array}$ & $\begin{array}{c}100 \mathrm{~cm} \mathrm{H}_{2} \mathrm{O}+ \\
10 \mathrm{~cm}^{1 / 10} \mathrm{n} . \mathrm{HCl}\end{array}$ & $\begin{array}{c}100 \mathrm{ccm}^{1 / 4} \mathrm{~m} . \\
\mathrm{Ca}\left(\mathrm{C}_{2} \mathrm{H}_{3} \mathrm{O}_{2}\right)_{2}+ \\
10 \mathrm{~cm} 1 / 10 \mathrm{n} . \mathrm{HCl}\end{array}$ & $\begin{array}{c}100 \mathrm{ccm} 1 / 4 \mathrm{~m} . \\
\mathrm{NaC}_{2} \mathrm{H}_{3} \mathrm{O}_{2}+ \\
10 \mathrm{~cm} 1 / 10 \mathrm{n} . \mathrm{HCl}\end{array}$ & $\begin{array}{c}100 \mathrm{~cm}^{1 / 4 \mathrm{~m}} . \\
\mathrm{KC}_{2} \mathrm{H}_{3} \mathrm{O}_{2}+ \\
10 \mathrm{~cm} 1 / 10 \mathrm{n} . \mathrm{HCl}\end{array}$ \\
\hline $\begin{array}{r}0 \\
2,40 \\
5,00 \\
15,15 \\
22,40 \\
45,00 \\
77,10 \\
101,50 \\
169,10\end{array}$ & 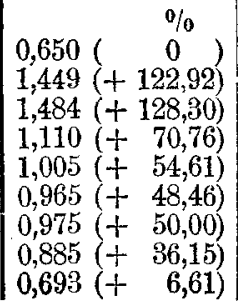 & 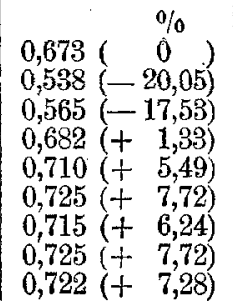 & $\begin{array}{l}0 \\
0,685\left(\begin{array}{c}\% \\
0\end{array}\right) \\
0,589(-14,01) \\
0,610(-10,94) \\
0,755(+10,21) \\
0,780(+13,86) \\
0,768(+12,11) \\
0,753(+9,92) \\
0,730(+6,56) \\
0,750(+9,48)\end{array}$ & $\begin{array}{l}0 \% \\
0,705\left(\begin{array}{c}0 \\
0\end{array}\right) \\
0,761(+7,94) \\
0,825(+17,02) \\
0,855(+21,27) \\
0,839(+19,00) \\
0,820(+16,31) \\
0,800(+13,47) \\
0,780(+10,63) \\
0,755(+7,09)\end{array}$ \\
\hline
\end{tabular}

Tabelle VI.

Laubfroschmuskeln. Beide Hinterbeine wurden bei jedem der Versuche benutzt.

\begin{tabular}{|c|c|c|c|c|}
\hline $\begin{array}{l}\text { Stunden } \\
\text { in der } \\
\text { Lösung }\end{array}$ & $\begin{array}{l}100 \mathrm{~cm} \mathrm{H}_{2} \mathrm{O}+ \\
10 \mathrm{~cm} 1 / 10 \mathrm{n} . \mathrm{HCl}\end{array}$ & $\begin{array}{c}100 \mathrm{ccm}^{1 / 2} \mathrm{~m} \\
\mathrm{Ca}\left(\mathrm{C}_{2} \mathrm{H}_{3} \mathrm{O}_{2}\right)_{2}+ \\
10 \mathrm{ccm}^{1 / 10} \mathrm{n} . \mathrm{HCl}\end{array}$ & $\begin{array}{c}100 \mathrm{~cm}{ }^{1 / 2 ~ m} . \\
\mathrm{NaC}_{2} \mathrm{H}_{3} \mathrm{O}_{2}+ \\
10 \mathrm{ccm}^{1 / 10} \mathrm{n} . \mathrm{HCl}\end{array}$ & $\begin{array}{c}100 \mathrm{ccm}^{1 / 2 \mathrm{~m}} . \\
\mathrm{KC}_{2} \mathrm{H}_{8} \mathrm{O}_{2}+ \\
10 \mathrm{ccm} 1 / 10 \mathrm{n} . \mathrm{HCl}\end{array}$ \\
\hline $\begin{array}{r}0 \\
2,30 \\
4,45 \\
15,00 \\
22,30 \\
44,45 \\
77,00 \\
101,40 \\
168,25\end{array}$ & 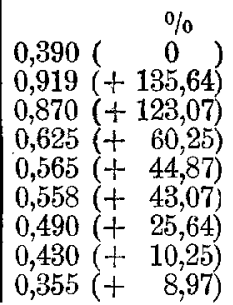 & $\begin{array}{l}0 \% \\
0,415\left(\begin{array}{c}0 \\
0\end{array}\right) \\
0,331(-20,24) \\
0,365(-12,03) \\
0,465(+12,04) \\
0,475(+14,45) \\
0,487(+17,34) \\
0,480(+15,66) \\
0,483(+16,38) \\
0,475(+14,45)\end{array}$ & $\begin{array}{l}0 \% \\
0,460\left(\begin{array}{c}0 \\
0\end{array}\right) \\
0,380(-17,39) \\
0,417(-9,34) \\
0,550(+19,56) \\
0,565(+22,82) \\
0,573(+24,56) \\
0,555(+20,65) \\
0,538(+16,95) \\
0,520(+13,04)\end{array}$ & 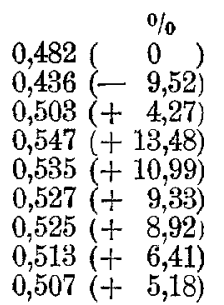 \\
\hline
\end{tabular}

Wie man sich durch einen Blick auf Fig. 7, 8, 9, 10 und 11 überzeugen kann, ist es nicht leicht, eine Tabelle aufzustellen, in der sich die verschiedenen Anion en nach ihrer Fähigkeit, die Quellung von Muskeln in Säurelösungen zu beeinflussen ordnen. Die einfachsten Beziehungen, von denen die Absorptionskurven für eine Reihe von Ammoniumsalzen wiedergegeben wurden, sind in Fig. $7 \mathrm{zu}$ finden. Es wurden jedesmal $10 \mathrm{ccm}$ einer 1/10 norm. Salzsäure $100 \mathrm{ccm}$ einer 1/4 molek. Lösung des entsprechenden Ammoniumsalzes zugesetzt. Die Anionen zeigten sich nach folgender Anordnung 
Über die Analogie zwischen der Wasserabsorption durch Fibrin etc. 85 wirksam. Die Quellung wird am wenigsten durch das erstgenannte Ion herabgesetzt.

$$
\frac{\mathrm{C}_{2} \mathrm{H}_{3} \mathrm{O}_{2}^{-}}{\text {(Acetat) }}, \frac{\mathrm{Cl}^{-}}{\text {(Chlorid) }}, \frac{\mathrm{NO}_{3}^{-}}{\text {(Nitrat) }}, \frac{\mathrm{SO}_{4}^{--}}{\text {(Sulphat) }}
$$

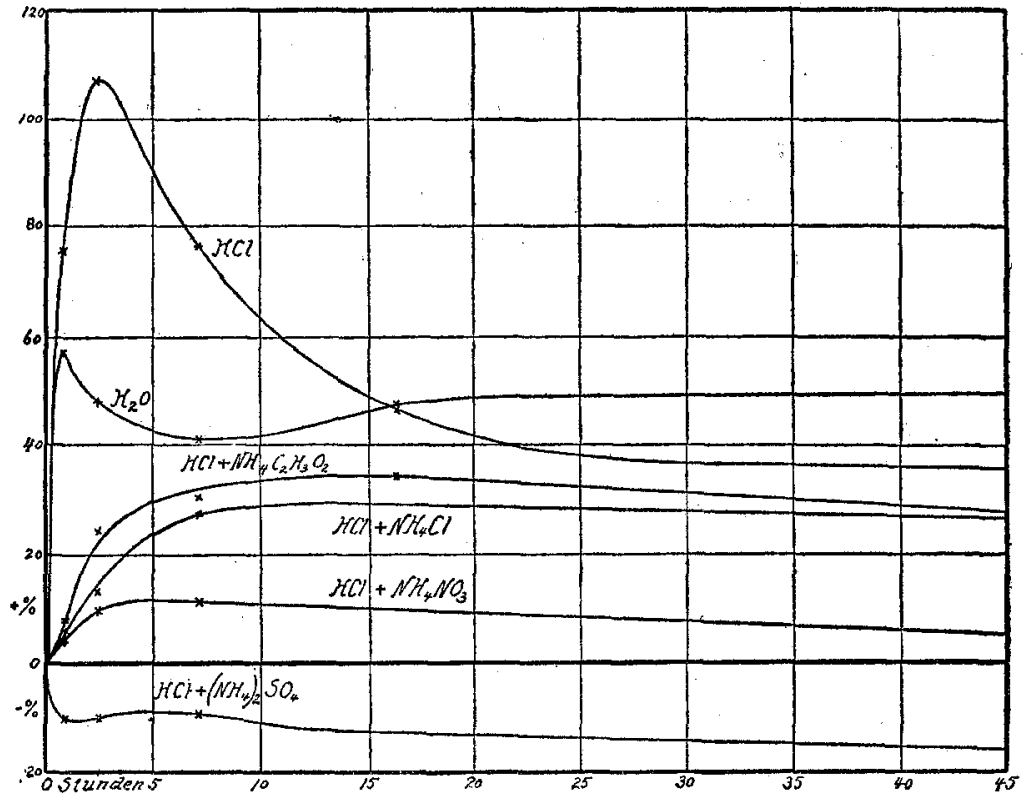

Fig. 7 .

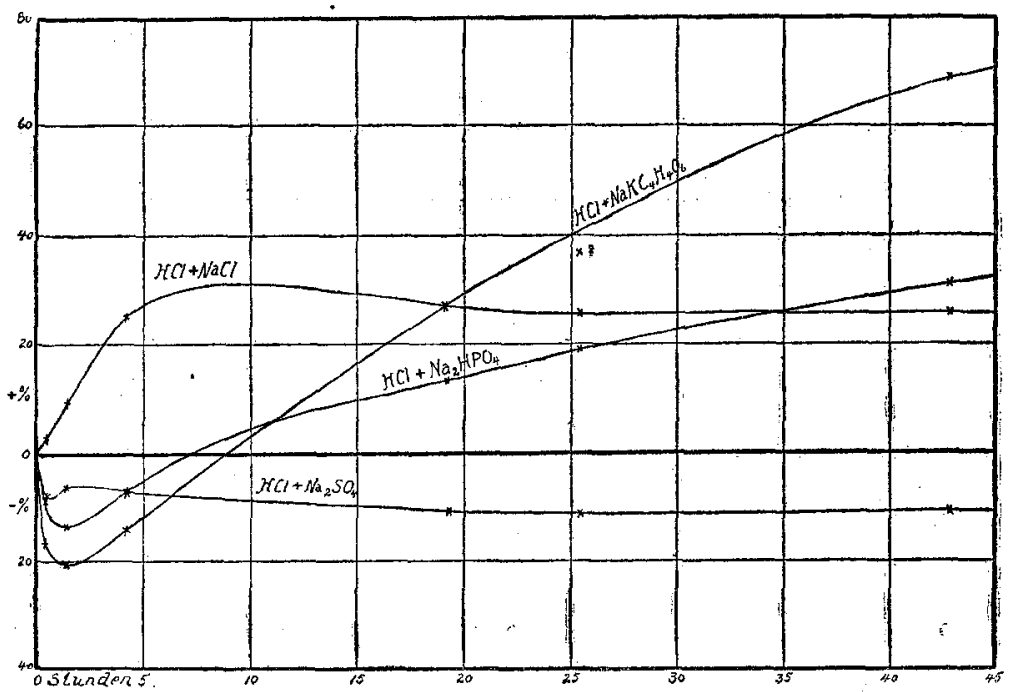

Fig. 8. 
Bei einer Anzahl Natriumsalze sind die Kurven noch komplizierter, was aus Fig. 8 zu ersehen ist. In den Anfangsversuchsstunden haben wir die Reihenfolge:

$$
\frac{\mathrm{Cl}^{-}}{\text {(Chlorid) }}, \frac{\mathrm{SO}_{4}^{--}}{\text {(Sulphat) }}, \frac{\left.\mathrm{HPO}_{4}--(?)^{1}\right)}{\text { (Phosphat) }}, \frac{\mathrm{C}_{4} \mathrm{H}_{4} \mathrm{O}_{6}--}{\text { ('Tartrat) }} \text {. }
$$

Die Anordnung der ersten zwei Ionen dieser Reihe stimmen mit der der Ammoniumreihe überein. Später finden wir jedoch diese Anọrdnung:

$$
\frac{\mathrm{C}_{4} \mathrm{H}_{4} \mathrm{O}_{6}-}{\text { (Tartrat) }}, \frac{\mathrm{HPO}_{4}^{--}}{\text {(Phosphat) }}, \frac{\mathrm{Cl}^{-}}{\text {(Chlorid)' }}, \frac{\mathrm{SO}_{4}^{--}}{\text {(Sulphat) }}
$$

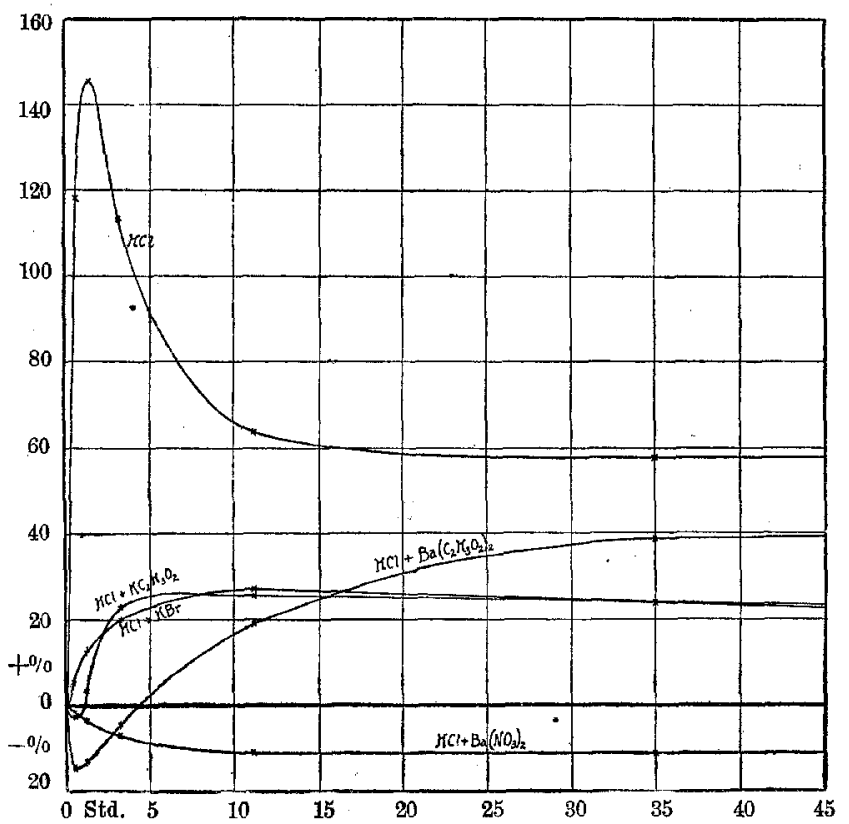

Fig. 9.

In Fig. 9 sind die durch Zusatz von $10 \mathrm{ccm}$ einer $1 / 10$ norm. Salzsäurelösung zu $100 \mathrm{cem}$ einer $1 / 4$ molek. Kaliumbromid-, Kaliumacetat-, Baryumacetat- oder Baryumnitratlösung erhaltenen Kurven wiedergegeben. Abgesehen von der anfänglichen Gewichtsabnahme jn der Kaliumacetatlösung laufen die zwei Kaliumsalzkurven ziemlich

1) Mit welchem Phosphorion wir es in diesen Versuchen zu tun haben, ist schwer zu bestimmen. 
Über die Analogie zwischen der Wasserabsorption durch Fibrin etc. 87 nah beieinander. Für die zwei Baryumsalze ist die Reihenfolge der Anionen (mit Ausnahme der ersten Versuchsstunden)

$$
\frac{\mathrm{C}_{2} \mathrm{H}_{3} \mathrm{O}_{2}^{-}}{\text {(Acetat) }}, \frac{\mathrm{NO}_{3}^{-}}{\text {(Nitrat) }}
$$

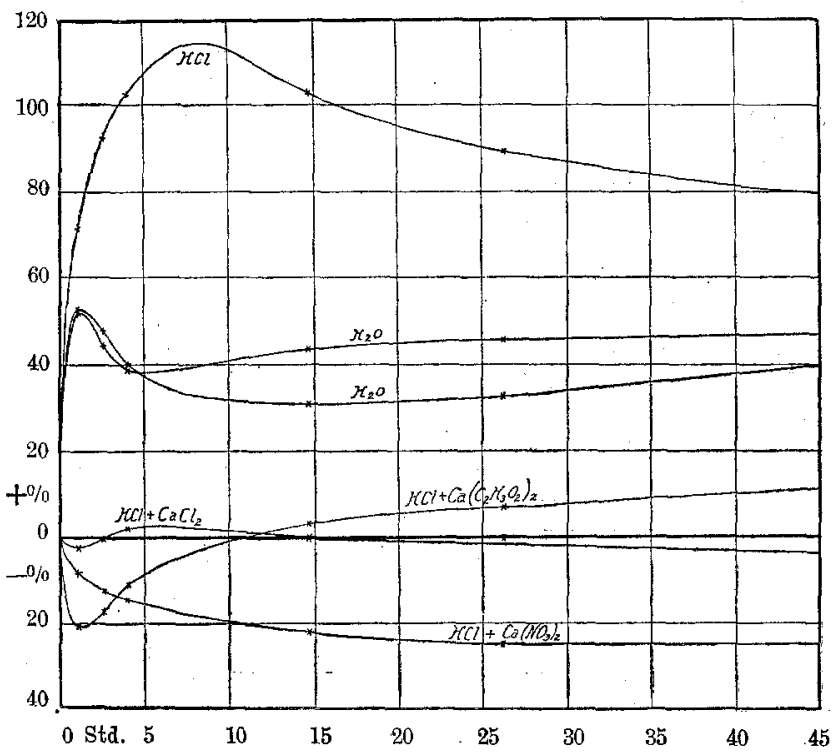

Fig. 10.

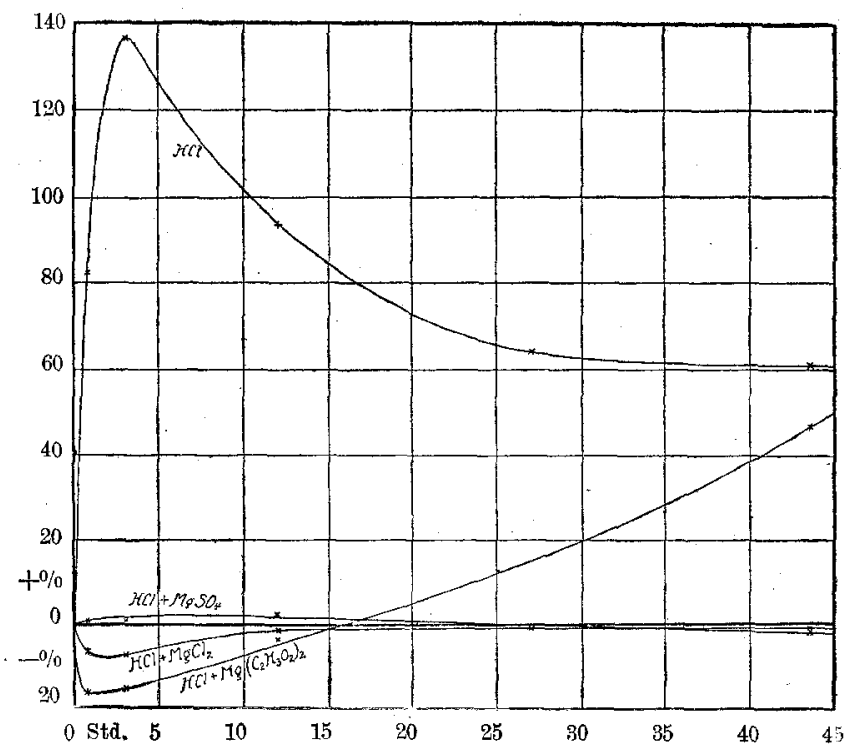

Fig. 11. 
Wenn $10 \mathrm{ccm}$ einer 1/10 normalen Salzsäure je $100 \mathrm{ccm}$ einer 1/4 molek. Calciumacetat-, Calciumchlorid- oder Calciumnitratlösung zugesetzt werden, erhält man die in Fig. 10 wiedergegebenen Kurven. Nach ungefähr zwölfstündigem Aufenthalt in den verschiedenen Lösungen finden wir wieder die schon bekannte Reihenfolge:

$$
\frac{\mathrm{C}_{2} \mathrm{H}_{3} \mathrm{O}_{2}^{-}}{\text {(Acetat) }}, \frac{\mathrm{Cl}^{-}}{\text {(Chlorid)' }}, \frac{\mathrm{NO}_{3}^{-}}{\text {(Nitrat) }} \text {. }
$$

Die Calciumacetatkurve stimmt mit der in Fig. 9 wiedergegebenen Baryumacetatkurve fast ganz überein; und die für Calciumnitrat mit der für Baryumnitrat.

In Fig. 11 finden wir die mit Magnesiumsalzen gewonnenen Resultate. Wie in den schon vorher beschriebenen Versuchen sind jedesmal $10 \mathrm{cem} 1 / 10$ norm. Salzsäure $100 \mathrm{cem}$ der entsprechenden 1/4 molek. Magnesiumsalzlösungen zugesetzt. Für die ersten Stunden des Versuchs zeigen sich die Anionen wie folgt geordnet:

$$
\frac{\mathrm{SO}_{4}--}{\text { (Sulphat) }}, \frac{\mathrm{Cl}^{-}}{\text {(Chlorid) }}, \frac{\mathrm{C}_{2} \mathrm{H}_{3} \mathrm{O}_{2}^{-}}{\text {(Acetat) }} \text {, }
$$

doch später finden wir die wohlbekannte Reihenfolge:

$$
\frac{\mathrm{C}_{2} \mathrm{H}_{3} \mathrm{O}_{2}^{-}}{\text {(Acetat) }}, \frac{\mathrm{Cl}^{-}}{\text {(Chlorid) }}, \frac{\mathrm{SO}_{4}^{--}}{\text {(Sulphat) }}
$$

Aus allen hier beschriebenen Kurven geht hervor, dass bei jedem Versuche, der die Wirkung eines Salzes definieren will, nicht allein bei Experimenten, die so ieinfach wie die hier geschilderten sind, sondern bei allen physiologischen Prozessen, bei welchen Salze eine Rolle spielen, das Zeitelement berücksichtigt werden muss. Wie aus den beschriebenen Kurvenreihen hervorgeht, kann ein Salz, das anfänglich einen wirklichen Gewichtsverlust bei einem in Säure befindlichen Muskel hervorruft, später die Quellung dieses Muskels reichlich gestatten. Dieser Erscheinung begegnet man z. B. bei den Acetaten und auch bei den bis jetzt untersuchten Tartraten und Phosphaten. Dies sind alle Salze "schwacher" Säuren, die in rein physikalisch-chemischer Hinsicht manche Besonderheiten aufweisen, - worauf wohl auch die besondere Wirkung dieser Salze bei verschiedenen physiologischen Erscheinungen zurückzuführen ist.

Es ist kein Versuch gemacht worden, die Unebenheiten mancher der Kurven zu erklären. Sie sind nicht zufällig. Gewichtsverschiedenheiten der verschiedenen Muskelmassen, Diffusionsunterschiede zwischen Salzen und Säuren, das Herausdiffundieren der Salze aus 
Über die Analogie zwischen der Wasserabsorption durch Fibrin etc.

den Muskeln in die umgebende Flüssigkeit, Verschiedenheiten in der Schnelligkeit, mit welcher das Gleichgewicht zwischen den organischen Bestandteilen der Muskeln mit der umgebenden Flüssigkeit hergestellt wird, und endlich Versehiedenheiten in der Schnelligkeit, mit welcher die Muskelkolloide mit den sie umgebenden anorganischen Bestandteilen ins Gleichgewicht kommen, sind Faktoren, die eine Rolle spielen, jedoch kann der Anteil eines jeden erst durch weitere Versuche bestimmt werden.

Die Tabellen VII, VIII, IX, $\mathrm{X}$ und XI enthalten die dureh Experiment bestimmten Gewichtsänderungen der Muskeln, aus welchen die resp. in Fig. 7, 8, 9, 10 und 11 gezeichneten Kurven gewonnen wurden.

\section{Tabelle VII.}

Laubfroscbmuskeln, Beide Hinterbeine wurden bei jeder der Lösungen benutzt.

\begin{tabular}{|c|c|c|c|}
\hline $\begin{array}{l}\text { Stunden in } \\
\text { der Lösung }\end{array}$ & $110 \mathrm{ccm} \mathrm{H}_{2} \mathrm{O}$ & $\begin{array}{c}100 \mathrm{~cm} \mathrm{H}_{2} \mathrm{O}+ \\
10 \mathrm{ccm} 1 / 10 \mathrm{n} . \mathrm{HCl}\end{array}$ & $\begin{array}{c}100 \mathrm{ccm} 1 / 4 \mathrm{~m} . \\
\mathrm{NH}_{4} \mathrm{C}_{2} \mathrm{H}_{3} \mathrm{O}_{2}+ \\
10 \mathrm{ccm} 1 / 10 \mathrm{n} . \mathrm{HCl}\end{array}$ \\
\hline $\begin{array}{r}0 \\
0,35 \\
2,20 \\
7,05 \\
16,35 \\
55,45 \\
72,20 \\
93,55 \\
146,50\end{array}$ & 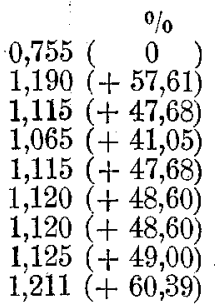 & 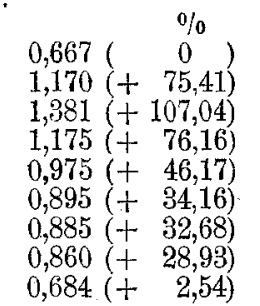 & 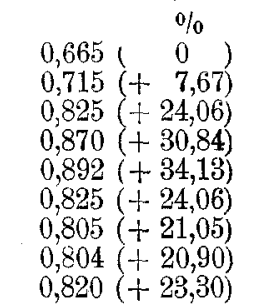 \\
\hline
\end{tabular}

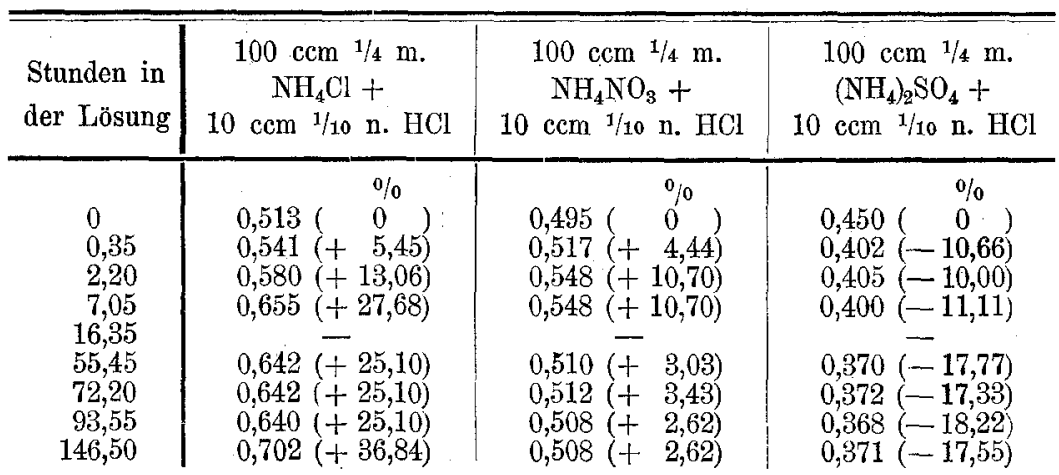

V.

Ein Muskel, der so viel Wasser als möglich in einer reinen Säurelösung absorbiert hat, quillt noch mehr, 
Tabelle VIII.

Laubfroschmuskeln. Beide Hinterbeine wurden bei jeder der Lösungen benutzt.

\begin{tabular}{|c|c|c|c|c|}
\hline $\begin{array}{l}\text { Stunden } \\
\text { in der } \\
\text { Lösung }\end{array}$ & $\begin{array}{c}100 \mathrm{~cm} 1 / 4 \mathrm{~m} \\
\mathrm{NaCl}+10 \mathrm{ccm} \\
1 / 10 \mathrm{n} . \mathrm{HCl}\end{array}$ & $\begin{array}{c}100 \mathrm{ccm}^{1 / 4} \mathrm{~m} . \\
\mathrm{Na}_{2} \mathrm{HPO}_{4}+ \\
10 \mathrm{ccm}^{1 / 10} \mathrm{n} . \mathrm{HCl}\end{array}$ & $\begin{array}{c}100 \mathrm{~cm} 1 / 4 \mathrm{~m} . \\
\mathrm{Na}_{2} \mathrm{SO}_{4}+10 \mathrm{ccm} \\
1 / 10 \mathrm{n} . \mathrm{HCl}\end{array}$ & $\begin{array}{c}100 \mathrm{~cm} 1 / 4 \mathrm{~m} . \\
\mathrm{NaKC} \mathrm{H}_{4} \mathrm{H}_{4} \mathrm{O}_{6}+ \\
10 \mathrm{ccm}^{1 / 10 \mathrm{n}} \text {. HCl }\end{array}$ \\
\hline $\begin{array}{r}0 \\
0,20 \\
1,20 \\
4,10 \\
19,10 \\
25,25 \\
42,45 \\
117,15\end{array}$ & 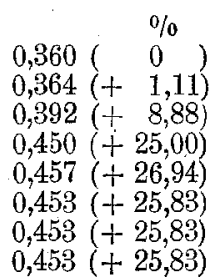 & $\begin{array}{l}0 \\
0,551\left(\begin{array}{c}0 \\
0\end{array}\right) \\
0,458(-16,87) \\
0,498(-20,50) \\
0,472(-14,33) \\
0,705(+27,94) \\
0,752(+36,47) \\
0,930(+68,78) \\
0,953(+72,95)\end{array}$ & $\begin{array}{l}\% \\
0,563\left(\begin{array}{c}0 \\
0\end{array}\right) \\
0,510(-9,41) \\
0,525(-6,74) \\
0,522(-7,28) \\
0,500(-11,18) \\
0,498(-11,54) \\
0,499(-11,36) \\
0,505(-10,30)\end{array}$ & 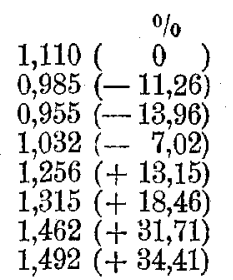 \\
\hline
\end{tabular}

\section{Tabelle IX.}

Laubfroschmuskeln. Beide Hinterbeine wưrden in jeder der Lösungen benutzt.

\begin{tabular}{|c|c|c|c|}
\hline $\begin{array}{l}\text { Stunden in } \\
\text { der Lösung }\end{array}$ & $\begin{array}{c}100 \mathrm{ccm} \mathrm{H}_{2} \mathrm{O}+ \\
10 \mathrm{~cm} 1 / 10 \mathrm{n} . \mathrm{HCl}\end{array}$ & $\begin{array}{c}100 \mathrm{ccm}^{1 / 4} \mathrm{~m} . \\
\mathrm{Ba}\left(\mathrm{C}_{2} \mathrm{H}_{3} \mathrm{O}_{2}\right)_{2}+ \\
10 \mathrm{ccm} 1 / 10 \mathrm{n} . \mathrm{HCl}\end{array}$ & $\begin{array}{c}100 \mathrm{ccm} 1 / 4 \mathrm{~m} . \\
\mathrm{Ba}\left(\mathrm{NO}_{3}\right)_{2}+10 \mathrm{ccm} \\
1 / 10 \mathrm{n} . \mathrm{HCl}\end{array}$ \\
\hline $\begin{array}{r}0 \\
0,35 \\
1,15 \\
3,10 \\
11,10 \\
35,00 \\
49,30 \\
75,00 \\
96,45\end{array}$ & 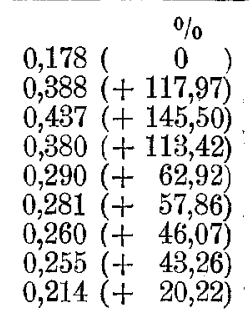 & $\begin{array}{c}\cdot \\
0,181\left(\begin{array}{c}\% \\
0\end{array}\right) \\
0,155(-14,36) \\
0,157(-13,25) \\
0,170(-5,11) \\
0,215(+18,78) \\
0,251(+32,62) \\
0,253(+39,78) \\
0,250(+38,12) \\
0,248(+37,02)\end{array}$ & 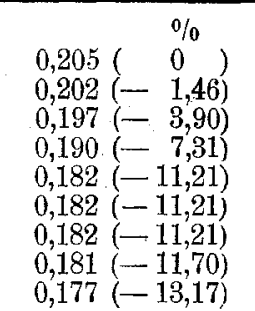 \\
\hline
\end{tabular}

\begin{tabular}{|c|c|c|}
\hline $\begin{array}{l}\text { Stunden in } \\
\text { der Lösung }\end{array}$ & $\begin{array}{c}100 \mathrm{~cm}{ }^{1 / 4} \mathrm{~m} . \mathrm{KC}_{2} \mathrm{H}_{3} \mathrm{O}_{2} \\
+10 \mathrm{~cm}{ }^{1 / 10 \mathrm{n}} . \mathrm{HCl}\end{array}$ & $\begin{array}{c}100 \mathrm{~cm} 1 / 4 . \mathrm{m} . \mathrm{KBr}+ \\
10 \mathrm{~cm} 1 / 10 \mathrm{n} . \mathrm{HCl}\end{array}$ \\
\hline $\begin{array}{r}0 \\
0,35 \\
1,15 \\
3,10 \\
11,10 \\
35,00 \\
49,30 \\
75,00 \\
96,45\end{array}$ & $\begin{array}{l}{ }^{0} / \\
0,229\left(\begin{array}{c}0 \\
0\end{array}\right) \\
0,222(-3,06) \\
0,235(+2,62) \\
0,280(+22,27) \\
0,298(+25,76) \\
0,284(+24,01) \\
0,278(+21,39) \\
0,267(+14,33) \\
0,265(+13,58)\end{array}$ & $\begin{array}{l}\% / \\
0,302\left(\begin{array}{c}0 \\
0\end{array}\right) \\
0,315(+4,30) \\
0,340(+12,58) \\
0,362(+19,86) \\
0,385(+27,48) \\
0,384(+23,84) \\
0,375(+24,17) \\
0,366(+21,19) \\
0,367(+21,85)\end{array}$ \\
\hline
\end{tabular}

wenn er in destilliertes Wasser gelegt wird. Diese Tatsache zeigt Fig. 12. Ein Gastrocnemius war in einer reinen Salz= säurelösung $\left(10 \mathrm{ccm} 1 / 10\right.$ norm. $\mathrm{HCl}+100 \mathrm{ccm} \mathrm{H}_{2} \mathrm{O}$ ) ad maximum gequollen und im Stadium des stetigen Gewichtsverlustes, als er in 
Über die Analogie zwischen der Wasserabsorption durch Fibrin etc.

\section{Tabelle X.}

Laubfroschmuskeln. Beide Hinterbeine wurden in jeder der Lösungen benutzt.

\begin{tabular}{|c|c|c|c|}
\hline $\begin{array}{l}\text { Stunden in } \\
\text { der Lösung }\end{array}$ & $110 \mathrm{ccm} \mathrm{H}_{2} \mathrm{O}$ & $110 \mathrm{ccm} \mathrm{H} \mathrm{H}_{2} \mathrm{O}$ & $\begin{array}{c}100 \mathrm{ccm} \mathrm{H}_{2} \mathrm{O}+ \\
10 \mathrm{ccm} 1 / 10 \mathrm{n} . \mathrm{HCl}\end{array}$ \\
\hline $\begin{array}{r}0 \\
1,00 \\
2,35 \\
4,05 \\
14,45 \\
26,25 \\
51,00 \\
77,40 \\
99,30 \\
125,00\end{array}$ & 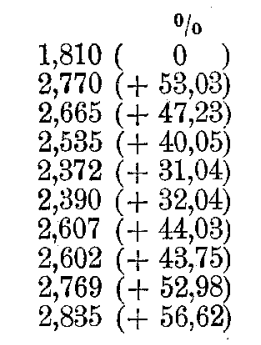 & 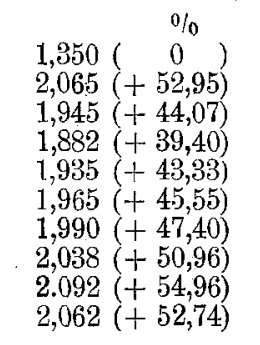 & $\begin{array}{c}0 / 0 \\
0,890\left(\begin{array}{c}0 \\
0\end{array}\right) \\
1,535(+72,47) \\
1,722(+93,48) \\
1,805(+102,80) \\
1,782(+100,22) \\
1,690(+89,88) \\
1,570(+76,40) \\
1,467(+64,83) \\
1,442(+62,02) \\
1,377(+54,71)\end{array}$ \\
\hline $\begin{array}{l}\text { Stunden in } \\
\text { der Lösung }\end{array}$ & $\begin{array}{c}100 \mathrm{ccm}^{1 / 4} \\
\mathrm{Ca}\left(\mathrm{C}_{2} \mathrm{H}_{3} \mathrm{O}_{2}\right)_{2}+ \\
10 \mathrm{ccm}^{1 / 10 \mathrm{n}} \mathrm{HCl}\end{array}$ & $\begin{array}{c}100 \mathrm{ccm} 1 / 4 \\
\mathrm{CaCl}_{2}+ \\
10 \mathrm{ccm} 1 / 10 \mathrm{n} . \mathrm{HCl}\end{array}$ & $\begin{array}{c}100 \mathrm{ccm} 1 / 4 \mathrm{~m} . \\
\mathrm{Ca}\left(\mathrm{NO}_{3}\right)_{2}+ \\
10 \mathrm{ccm} 1 / 10 \mathrm{n} . \mathrm{HCl}\end{array}$ \\
\hline $\begin{array}{r}0 \\
1,00 \\
2,35 \\
4,05 \\
14,45 \\
26,25 \\
51,00 \\
77,40 \\
99,30 \\
125,00\end{array}$ & $\begin{array}{l} \\
0,490\left(\begin{array}{c}0 \\
0\end{array}\right) \\
0,390(-20,40) \\
0,405(-17,34) \\
0,435(-11,22) \\
0,505(+3,06) \\
0,522(+6,53) \\
0,552(+12,65) \\
0,548(+11,83) \\
0,552(+12,65) \\
0,547(+11,63)\end{array}$ & $\begin{array}{l}0 / 0 \\
0,410\left(\begin{array}{c}0 \\
0\end{array}\right) \\
0,392(-2,92) \\
0,408(-0,48) \\
0,417(+1,70) \\
0,410(0) \\
0,410(0) \\
0,390(-4,87) \\
0,391(-4,63) \\
0,386(-5,87) \\
0,378(-7,80)\end{array}$ & $\begin{array}{l}0 \\
0,410\left(\begin{array}{c}\% \\
0\end{array}\right) \\
0,375(-8,53) \\
0,360(-12,19) \\
0,350(-14,63) \\
0,322(-21,46) \\
0,310(-24,39) \\
0,311(-24,14) \\
0,301(-26,58) \\
0,307(-25,12) \\
0,302(-26,34)\end{array}$ \\
\hline
\end{tabular}

Tabelle XI.

Laubfroschmuskeln. Beide Hinterbeine wurden in jeder der Lösungen benutzt.

\begin{tabular}{|c|c|c|c|c|}
\hline $\begin{array}{c}\text { Stunden } \\
\text { in der } \\
\text { Lösung }\end{array}$ & $\begin{array}{c}100 \mathrm{~cm} \mathrm{H}_{2} \mathrm{O}+ \\
10 \mathrm{ccm}^{1 / 10 \mathrm{n}} \mathrm{HCl}\end{array}$ & $\begin{array}{c}100 \mathrm{ccm}^{1 / 4} \mathrm{~m} . \\
\mathrm{Mg}\left(\mathrm{C}_{2} \mathrm{H}_{3} \mathrm{O}_{2}\right)_{2}+ \\
10 \mathrm{~cm}^{1 / 10 ~ \mathrm{n}} \mathrm{HCl}\end{array}$ & $\begin{array}{c}100 \mathrm{ccm} 1 / 4 \mathrm{~m} . \\
\mathrm{MgCl}_{2}+10 \mathrm{ccm} \\
1 / 10 \mathrm{n} . \mathrm{HCl}\end{array}$ & $\begin{array}{c}100 \mathrm{ccm} 1 / 4 \mathrm{~m} . \\
\mathrm{MgSO}_{4}+10 \mathrm{ccm} \\
1 / 10 \mathrm{HI} . \mathrm{HCl}\end{array}$ \\
\hline $\begin{array}{r}0 \\
0,40 \\
3,00 \\
12,00 \\
27,00 \\
43,30 \\
65,30 \\
122,15\end{array}$ & \begin{tabular}{|c|c|} 
& 0 \\
0,585 & $\left(\begin{array}{c}0 \\
1,065\end{array}\right)$ \\
$1,065(+$ & $82,05)$ \\
$1,383(+$ & $136,41)$ \\
$1,132(+$ & $98,50)$ \\
$0,960(+$ & $64,10)$ \\
$0,940(+$ & $60,68)$ \\
$0,882(+$ & $50,76)$ \\
$0,655(+$ & $11,96)$
\end{tabular} \mid & 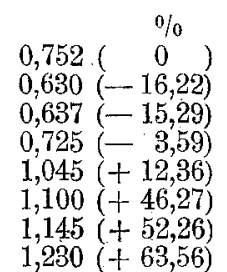 & $\begin{array}{l}0 \% \\
0,780\left(\begin{array}{c}0 \\
0,728\end{array}\right) \\
0,720(-6,66) \\
0,790(-1,69) \\
0,774(-0,76) \\
0,767(-1,66) \\
0,748(-4,10) \\
0,754(-3,33)\end{array}$ & $\begin{array}{l}0 \% \\
0,955\left(0^{0}\right) \\
0,964(+0,94) \\
0,963(+0,83) \\
0,975(+2,09) \\
0,943(-1,25) \\
0,945(-1,04) \\
0,925(-3,15) \\
0,935(-2,09)\end{array}$ \\
\hline
\end{tabular}


destilliertes Wasser versetzt wurde. Die erhöhte Wasserabsorption nach dieser Versetzung kommt durch den plötzlichen Richtungswechsel in der Kurve $A, A^{\prime}, A^{\prime \prime}$ klar zum Vorschein.

Die Übertragung aus der einen Lösung in die andere ist durch einen Pfeil angedeutet. Bei unseren Versuchen über die Quellung des Fibrins fanden Gertrude Moore und ich ein äbnliches Verhalten, denn Fibrin, das so viel als möglich in einer Säurelösung

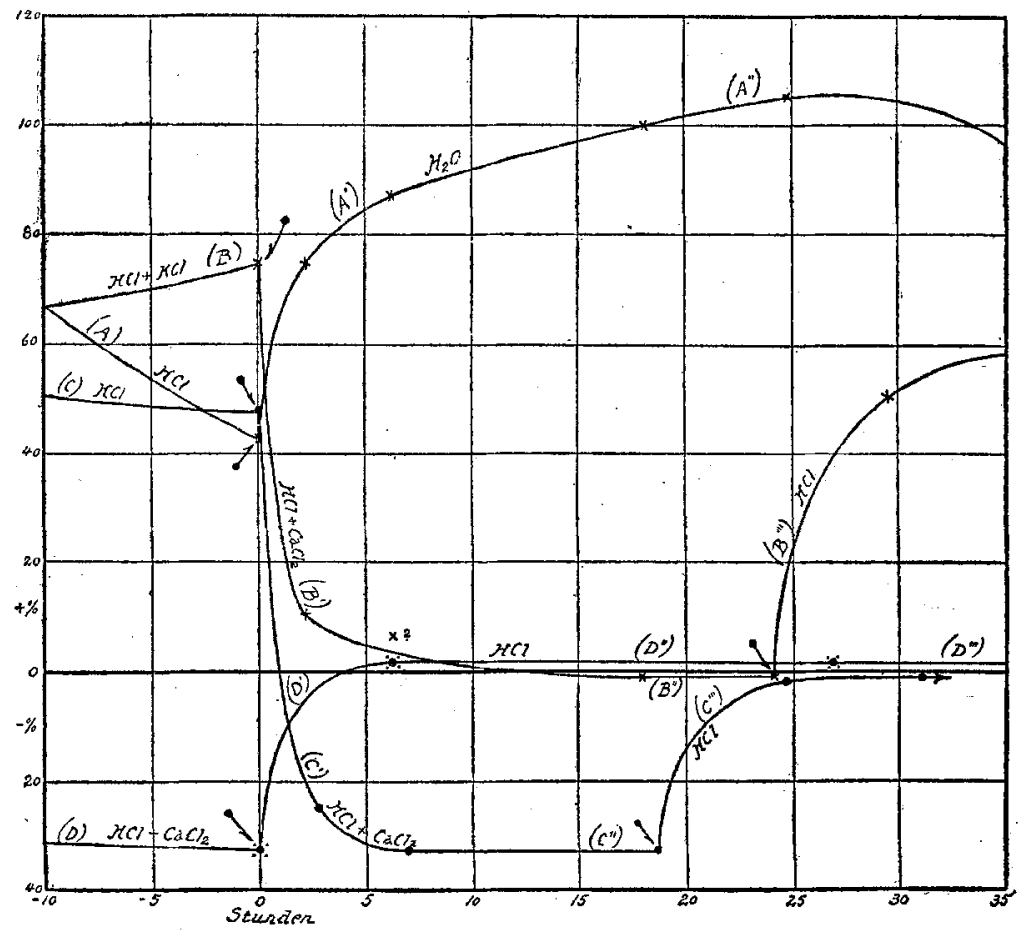

Fig. 12.

gequollen ist, nimmt nach Versetzung in destilliertes Wasser noch mehr davon auf $\left.{ }^{1}\right)$. Der erste Teil der Kurve $A, A^{\prime}, A^{\prime \prime}$ ist eine Fortsetzung der in Fig. 3 aufgezeichneten niedrigen Salzsäurekurve.

1) Bei Fibrinversuchen ist dieser Satz nur in dem Falle richtig, wenn die optimale Konzentration der Säure für die Quellung des Fibrins überschritten worden ist. Aller Wahrscheinlichkeit nach ist bei diesen Versuchen mit Muskel die optimale Konzentration der Säure für die Quellung auch überschritten, denn in hier nicht mitgeteilten Versuchen, bei welchen höhere HCl-Konzentrationen benutzt wurden als in den hier beschriebenen, bemerkte ich keine grössere Quellung, sondern eine verminderte. 
Uber die Analogie zwischen der Wasserabsorption durch Fibrin etc. 93

Die ersten in dem Muskel beohachteten Gewichtsänderungen sind in Tabelle $12 \mathrm{zu}$ finden. Nachfolgend sind die Gewichtsänderungen, die nach Versetzung aus der reinen Säurelösung in destilliertes Wasser beobachtet wurden, angegeben. Aus diesen ist die in Fig. 12 gefundene Kurve konstruiert.

Der benutzte Gastrocnemius wog ursprünglich in Gramm

$$
1,12(0 \%) \text {. }
$$

Nach Aufenthalt während der angegebenen Stunden in $10 \mathrm{ccm}$ $1 / 10$ norm. $\mathrm{HCl}+100$ cem $\mathrm{H}_{2} \mathrm{O}$ stieg das Gewicht.

$\begin{array}{ccc}\text { Stunden } & \text { Gramm } & \% \\ 28,30 & 2,31 & (+106,25) \\ 52,50 & 1,60 & (+42,84)\end{array}$

Hier wurde der Muskel in Wasser versetzt. Nach diesem Punkt wurden die Stunden von neuem gezählt.

$\begin{array}{rrr}2,00 & 1,95 & (+74,10) \\ 6,10 & 2,10 & (+87,50) \\ 18,10 & 2,24 & (+100,00) \\ 24,40 & 2,30 & (+105,35) \\ 38,40 & 2,07 & (+84,82)\end{array}$

VI.

Die Wasserabsorption und Wassersekretion durch Muskel stellt im hohen Maasse einen umkehrbaren Prozess dar. Der Prozess ist jedoch nicht (innerhalb der begrenzten Versuchszeiten) vollständig umkehrbar, denn durch Behandlung mit irgendeiner Lösung scheint ein mehr oder minder anhaltender Eindruck auf den Muskel ausgeübt zu werden, der auch eine Zeitlang nach Wiederherstellung des ursprünglichen Zustandes anhält. Die in Fig. 12 gezeichneten Kurven stellen diese Tatsachen graphisch dar. Versetzung aus einer Lösung in eine andere ist jedesmal durch einen Pfeil angedeutet.

In der Kurve $C, C^{\prime}, C^{\prime \prime}, C^{\prime \prime \prime}$ ist der Effekt einer Versetzung aus reiner Salzsäurelösung in eine solche, die Calciumchlorid enthält und dann eine zweite Rückversetzung in die erste Lösung dargestellt. Die auffallende Volumsverminderung (Entquellung) nach Versetzung in die Calciumchlorid enthaltende Salzsäurelösung ist durch den plötzlichen Richtungswechsel der Kurve leicht zu bemerken. Wie unvollständig dieser Gewichtsverlust in der reinen Salzsäurelösung 
wieder gutgemacht wird, zeig'en die letzten Teile der Kurve: Die ersten an dem Muskel beobachteten Gewichtsänderungen sind in der ersten Spalte der Tabelle III angegeben und in der hohen Salzsäurekurve der Fig. 3. Die in Fig. 12 sich befindende Kurve ist aus den folgenden Versuchseinzelheiten gewonnen. Ursprünglich wog der Muskel in Gramm $\quad 0,75 \quad(0 \%)$.

Nach dem Verlauf der hier angegebenen Stunden in $10 \mathrm{ccm}$ $1 / 10$ n. $\mathrm{HCl}+100$ cem $\mathrm{H}_{2} \mathrm{O}$ wog der Muskel

$\begin{array}{ccc}\text { Stunden } & \text { Gramm } & \% \\ 44,40 & 1,22 & (+62,66) \\ 89,10 & 1,11 & (+48,00)\end{array}$

Um diese Zeit war der Muskel zu einer weichen Masse gequollen und konnte nur schwer gehandhaht werden. Die äusseren Muskelteile waren klar und gelatinös und blieben an dem Filtrierpapier, mit denen sie in Berührung kamen, kleben. Der Muskel wurde hierauf in eine Lösung, bestehend aus $10 \mathrm{ccm} 1 / 10$ norm. $\mathrm{HCl}+100 \mathrm{~cm}{ }^{1 / 2}$ molek. $\mathrm{CaCl}_{2}$, getan. In dieser wurde er wieder fest, nichtklebrig, und konnte leicht gehandhabt werden. Die Gewichtsänderungen waren wie folgt. Die Stunden wurden von Zeit der Versetzung von neuem gezählt.

$\begin{array}{ccc}\text { Stunden } & \text { Gramm } & \mathbf{0} \% \\ 0,45 & 0,80 & (+6,66) \\ 2,45 & 0,56 & (-25,33) \\ 6,55 & 0,51 & (-32,00) \\ 18,45 & 0,51 & (-32,00)\end{array}$

Rückversetzung in die ursprüngliche Salzsäurelösung ergab folgendes Resultat:

$$
\begin{array}{lll}
24,45 & 0,74 & (-1,33) \\
31,00 & 0,74 & (-1,33)
\end{array}
$$

Eine Kurve, die in vielen Beziehungen der eben beschriebenen gleich ist, ist durch $B, B^{\prime}, B^{\prime \prime}, B^{\prime \prime \prime}$ in Fig. 12 angedeutet. Sie zeigt den Effekt der Versetzung eines Muskels aus einer Chlorkali enthaltenden Salzsäurelösung in eine Cblorcalcium enthaltende und dann eine zweite Versetzung in eine reine Salzsäurelösung. Die ersten Stunden des Versuchs sind graphisch hier nicht wiedergegeben. Die untenstehenden Zahlen zeigen jedoch klar, dass in der Chlorkali enthaltenden Salzsäure der Muskel an Gewicht stetig zunabm. Versetzung in eine Chlorcalcium enthaltende Säurelösung wurde 
durch einen prompten Gewichtsverlust gekennzeichnet ${ }^{1}$ ). Eine Gewichtszunabme wurde nnach Versetzung aus dieser Lösung in eine reine Salzsäurelösung bemerkt, doch war wieder die Gewichtszunahme nicht genügend gross, um selbst den höchsten der in der $\mathrm{HCl}-\mathrm{KCl}-$ Lösung erhaltenen Punkt zu erreichen. Die Kurve ist aus den folgenden Wägungen konstruiert. Ursprünglich wog der Muskel in Gramm

$$
1,14 \quad(0 \%)
$$

Nach den hier angegebenen Stunden in $10 \mathrm{ccm} 1 / 10$ norm. $\mathrm{HCl}+100 \mathrm{cem} \mathrm{1/8} \mathrm{molek.} \mathrm{KCl}$ änderte sich das Gewicht wie folgt:

$\begin{array}{ccc}\text { Stunden } & \text { Gramm } & \% \\ 2,50 & 1,52 & (+33,33) \\ 4,50 & 1,70 & (+49,12) \\ 9,50 & 1,87 & (+64,03) \\ 27,55 & 1,86 & (+63,15) \\ 52,20 & 2,00 & (+75,43)\end{array}$

Hierauf wurde der Muskel in $10 \mathrm{ccm} 1 / 10$ norm. $\mathrm{HCl}+100 \mathrm{ccm}$ $1 / 2$ molek. $\mathrm{CaCl}_{2}$ versetzt. Die Stunden wurden von neuem gezählt.

$\begin{array}{rrr}2,00 & 1,36 & (+10,52) \\ 6,10 & 1,21 & (+6,14) \\ 18,00 & 1,12 & (-1,75) \\ 24,00 & 1,12 & (-1,75)\end{array}$

Dann wurde der Muskel in $10 \mathrm{cem} 1 / 10$ norm. $\mathrm{HCl}+100 \mathrm{cem} \mathrm{H}_{2} \mathrm{O}$ eingebracht.

$$
\begin{array}{lll}
29,25 & 1,72 & (+50,87) \\
50,30 & 1,79 & (+57,01)
\end{array}
$$

Die Kurve $D, D^{\prime}, D^{\prime \prime}, D^{\prime \prime \prime}$ der Fig. 12 zeigt die Gewichtszunahme eines Muskels nach Versetzung aus einer Calciumchlorid enthaltenden Salzsäurelösung in eine reine Säurelösung von derselben Konzentration. Die Gewichtsänderungen des Muskels während der ersten Versuchsstunden in dem $\mathrm{HCl}-\mathrm{CaCl}_{2}$-Gemisch sind in der zweiten Spalte der Tabelle III wiedergegeben und in der niedrigsten Kurve der Fig. 3 aufgezeichnet. Ursprünglich wog der Muskel in Gramm $0,77 \quad(0 \%)$

1) Eigentlich hätten isosmotische Kaliumchlorid- und Calciumchloridlösungen benutzt werden sollen, denn ein Gewichtsverlust wäre auch in dem Falle, wenn von einer niedrigen zu einer höheren Kaliumchloridkonzentration übergegangen wäre, eingetreten. Doch wäre er unter diesen Umständen geringer gewesen. Siehe Fig. 2 u. 3. . 
Nach Aufenthalt in $10 \mathrm{cem} 1 / 10$ norm. $\mathrm{HCl}+100 \mathrm{~cm} \mathrm{1/2} \mathrm{molek.}$ $\mathrm{CaCl}_{2}$ ergaben sich folgende Werte:

$\begin{array}{rcc}\text { Stunden } & \text { Gramm } & \% \\ 89,10 & 0,53 & (-31,16) \\ 114,00 & 0,52 & (-32,47) .\end{array}$

Hier fand die Versetzung in $10 \mathrm{~cm} 1 / 10$ norm. $\mathrm{HCl}+100 \mathrm{ccm}$ $\mathrm{H}_{2} \mathrm{O}$ statt. Die Stunden wurden von neuem gezählt.

$\begin{array}{rll}6,10 & 0,79 & (+2,59) \\ 26,40 & 0,79 & (+2,59) \\ 71,10 & 0,78^{\circ} & (+1,29)\end{array}$

Aus diesen Beobachtungen geht klar hervor, dass ein ziemlich anhaltender Einfluss auf einen Muskel durch jeden Lösungswechsel ausgeübt wird. Aus der letzt beschriebenen Kurve ist zu ersehen, dass vorheriger Aufentbalt in einer Calciumchlorid enthaltenden Lösung den Muskel verbindert, in einer reinen Salzsäurelösung genügend wieder aufzuquellen, um auch nur annähernd den ursprünglich möglichen Quellungsgrad zu erreichen.

\section{VII.}

Nichtelektrolyte teilen nicht die bei Elektrolyten ausgesprochene Fähigkeit die Wasserabsorption durch Muskeln in Säurelösungen zu beeinflussen. Ein Blick auf Fig. 3 und 13 beweist dies. Bei Fig. 3 kann der Effekt eines Harnstoffzusatzes mit dem eines von Calciumchlorid zu einer reinen Salzsäurelösung verglichen werden. Während eine Gewichtsänderung in einer Lösung von $10 \mathrm{cem} 1 / 10$ norm. $\mathrm{HCl}+100 \mathrm{ccm} 1 / 4$ molek. $\mathrm{CaCl}_{2}$ kaum stattfindet, nimmt eine Muskel in $10 \mathrm{~cm}^{1 / 10}$ norm. $\mathrm{HCl}+100 \mathrm{cem}{ }^{1 / 2}$ molek. Harnstoff stetig an Gewicht zu, so dass er am Ende des Experiments ebensoviel wägen kann, als wäre er in eine reine Salzsäurelösung eingebracht. Diese Quellungsverschiedenheit des Muskels in den zwei Lösungen ist nicht auf osmotische Unterschiede zurückzuführen. Der osmotische Druck einer $1 / 2$ molekularen Harnstoff lösung ist der einer 1/4 molekularen Calciumchloridlösung ungefähr gleich. Oder um noch einen stärkeren Beweis zu erlangen, ist es nur nötig, die durch Gebrauch der 1/8 molekularen Calciumchloridlösung gewonnene Kurve mit der durch Benutzung der osmotisch viel stärkeren $1 / 2$ molekularen Harnstofflösung zu vergleichen. Die zwei Harnstoffkurven und die niedrige Salzsäurekurve, welche in Fig. 3 aufgezeichnet sind, wurden aus den in Tabelle XII 
Über die Analogie zwischen der Wasserabsorption durch Fibrin etc.

wiedergegebenen Gewichtsbestimmungen konstruiert. Die Gewichtsbestimmungen für die hohe Salzsäurekurve und die drei Calciumchloridkurven sind in Tabelle III zu finden.

Die Kurven der Fig. 13 liefern einen weiteren Beweis dafür, dass Nichtelektrolyte die ausgesprochene Fähigkeit, die Wasser-

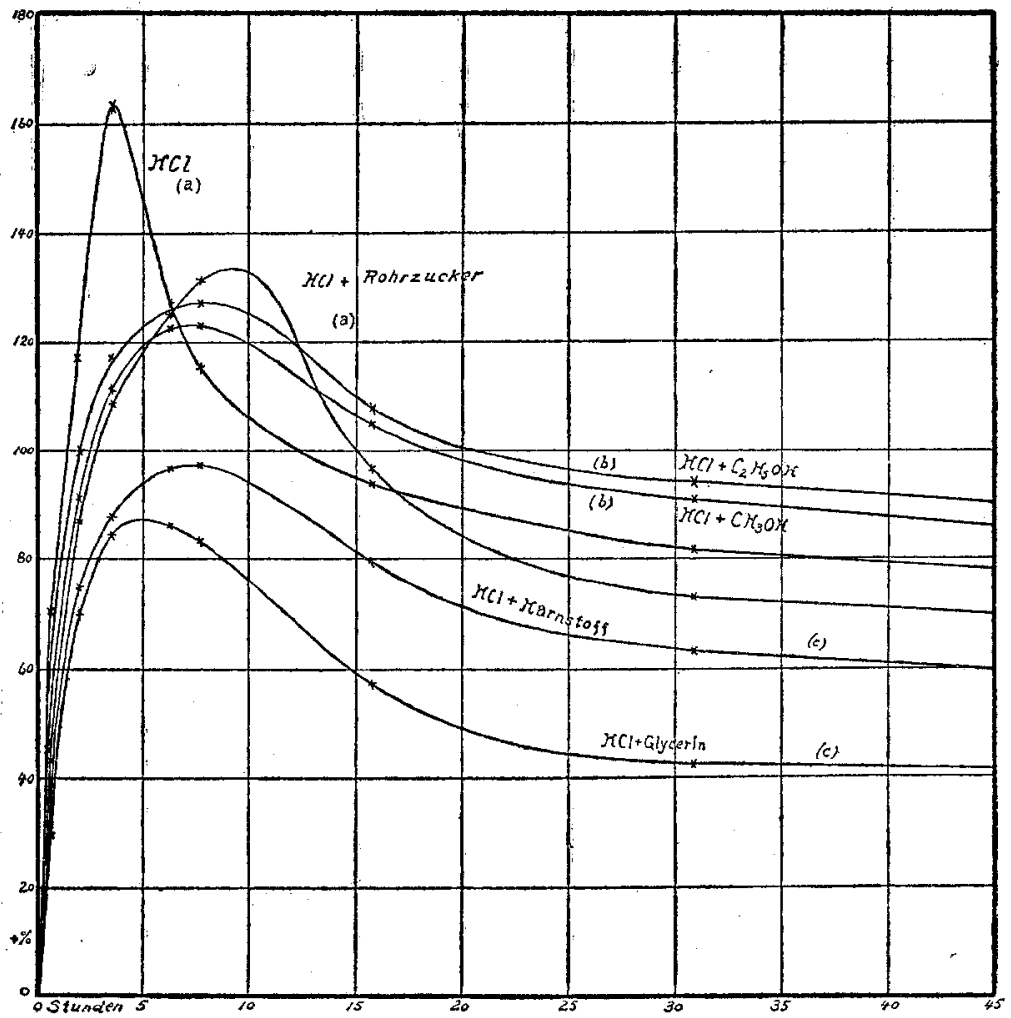

Fig. 13.

absorption durch Muskeln in Säurelösungen zu beeinflussen, mit Elektrolyten nicht teilen. Die Kurven sind aus Beobachtungen über die Quellung von Laubfroschhinterbeinen konstruiert und können mit irgendeiner der vorhergegangenen Kurven verglichen werden, mit Ausnahme derjenigen, die sich auf Gastrocnemiusmuskeln.allein beziehen (Fig. 2, 3, 12). Jede Lösung wurde durch Zusatz von $10 \mathrm{cem}{ }^{1 / 10}$ norm. Salzsäure zu $100 \mathrm{ccm}$ der entsprechenden $1 / 2$ molek. Lösung eines Nichtelektrolytes bereitet. Man sieht sofort, dass nicht einer der Nichtelektrolyte imstande ist, die Quellung der verschiedenen Muskeln auch nur in annähernder Weise so stark wie E. Pflù ger, Archiv für Physiologie. Bd. 124. 
Tabelle XII.

Gastrocnemiusmuskeln. Die Muskeln in den Harnstofflösungen stammen von demselben Frosch.

\begin{tabular}{|c|c|c|c|}
\hline $\begin{array}{l}\text { Stunden } \\
\text { in } \\
\text { der Lösung }\end{array}$ & $\begin{array}{c}100 \mathrm{ccm} \mathrm{H}_{2} \mathrm{O}+ \\
10 \mathrm{ccm} 1 / 10 \mathrm{n} . \mathrm{HCl}\end{array}$ & $\begin{array}{c}100 \mathrm{~cm} 1 / 2 \mathrm{~m} . \text { Harn- } \\
\text { stoff }+10 \mathrm{ccm} 1 / 10 \mathrm{n} . \\
\mathrm{HCl}\end{array}$ & $\begin{array}{c}100 \mathrm{ccm}^{1 / 4} \mathrm{~m} . \text { Harn- } \\
\text { stoff }+10 \mathrm{ccm} 1 / 10 \mathrm{n} . \\
\mathrm{HCl}\end{array}$ \\
\hline $\begin{array}{r}0 \\
2,50 \\
4,50 \\
8,35 \\
28,20 \\
52,50\end{array}$ & $\begin{array}{l}{ }^{0 / 0} \\
1,12\left(0^{2}\right) \\
2,25(+100,89) \\
2,50(+128,21) \\
2,69(+140,17) \\
2,31(+106,25) \\
1,60(+42,84)\end{array}$ & $\begin{array}{l}0 \% \\
0,86\left(0^{0 / 0}\right) \\
1,04(+20,98) \\
1,15(+33,72) \\
1,40(+62,79) \\
2,05(+138,37) \\
2,25(+161,62)\end{array}$ & $\begin{array}{l}{ }^{0 / 0} \\
0,82\left({ }^{0}\right) \\
1,25(+52,43) \\
1,46(+78,04) \\
1,69(+106,09) \\
1,88(+128,17) \\
1,64(+100,00)\end{array}$ \\
\hline
\end{tabular}

isosmotische Lösungen der Elektrolyte zu hemmen. Die allgemeine Form der Kurven, welche die Gewichtsänderungen in reinen Salzsäurelösungen und in solchen, denen ein Nichtelektrolyt zugesetzt ist, veranschaulichen, ist in beiden Fällen ungefähr dieselbe. Nur ein Laubfroschhinterbein wurde in jeder der Lösungen bei dieser Versuchsreibe benutzt. Bei $(a)(a),(b)(b)$ und $(c)(c)$ können die Glieder desselben Beinpaares bemerkt werden, und man sieht, dass die Kurven, welche die Quellung jedes der Glieder eines Beinpaares darstellen, sehr nahe zusammen liegen. Dies deutet darauf hin, dass die Verschiedenheiten bei der ganzen Kurvenreihe mehr auf individuellen Unterschieden der Muskeln beruhen als auf wirklichen Unterschieden zwischen der Wirkung der verschiedenen Nichtelektrolyte. Doch soll hiermit nicht gesagt sein; dass Nichtelektrolyte absolut unfähig sind, die Quellung von Muskeln in Säurelösungen zu beeinflussen. Zurzeit bin ich zufrieden, festzustellen, dass bei gleicher osmotischer Konzentration die Elektrolyte den Nichtelektrolyten in Quellungshemmender Wirkung weit überlegen sind.

Wird die in Fig. 13 gezeichnete Harnstoffkurve mit der in Fig. 3 verglichen, so bemerkt man, dass die bei Fig. 3 benutzten Harnstofflösungen scheinbar stärker die Quellungskurve beeinflussen als die in Fig. 13. Die in Fig. 13 befindliche Kurve ist durch Gebrauch einer trischpräparierten Harnstofflösung gewonnen und stellt deshalb die Wirkung von Harnstoff prägnanter dar als die in Fig. 3. Die zu Fig. 3 benutzten Harnstofflösungen hatten eine Zeitlang gestanden und enthielten wahrscheinlich Elektrolyte (Ammoniumverbindungen).

Dieser wesentliche Unterschied zwischen der Wirkung von 
Elektrolyten und Nichtelektrolyten auf die Quellung eines Kolloids ist wohl nicht ohne physiologisches Interesse. Doch können Einzelheiten hier nicht diskutiert werden. Genüge es, die Aufmerksamkeit auf die vielen physiologischen Erscheinungen zu lenken (Wachstum, Sekretion, Muskel- und Nervenaktivität), in welchen Elektrolyte eine grosse Rolle spielen und Nichtelektrolyte relativ wirkungslos sind. Wo soleh ein Unterschied existiert, tun wir wohl jedesmal wohl daran, den Grund der Erscheinung in Änderungen der Gewebekolloide zu suchen.

Die folgende Tabelle XIII entbält die Versuchsdaten, aus denen die in Fig. 13 sich befindenden Kurven aufgebaut wurden.

\section{Tabelle XIII.}

Laubfroschmuskeln. Ein Hinterbein wurde in jeder der Lösungen benutzt. Glieder desselben Paars Beine können durch denselben Buchstaben erkannt werden.

\begin{tabular}{|c|c|c|c|}
\hline $\begin{array}{l}\text { Stunden } \\
\text { in } \\
\text { der Lösung }\end{array}$ & $\begin{array}{c}100 \mathrm{ccm} 1 / 2 \mathrm{~m} . \text { Athyl. } \\
\text { alkohol }+10 \mathrm{ccm} \\
1 / 10 \mathrm{n} . \mathrm{HCl}\end{array}$ & $\begin{array}{c}100 \mathrm{ccm}^{1 / 2 \mathrm{~m}} \text {. Methyl- } \\
\text { alkohol }+10 \mathrm{ccm} \\
1 / 10 \text { n. HCl }\end{array}$ & $\begin{array}{c}100 \mathrm{ccm} 1 / 2 \mathrm{~m} . \\
\text { Glycerin }+10 \mathrm{ccm} \\
1 / 10 \mathrm{n} . \mathrm{HCl}\end{array}$ \\
\hline $\begin{array}{r}0 \\
0,33 \\
1,52 \\
3,25 \\
6,15 \\
7,40 \\
15,40 \\
30,55 \\
54,10\end{array}$ & $\begin{array}{c}\% \\
0,402\left(\begin{array}{c}0 \\
0,402\end{array}\right) \\
0,630(+56,71) \\
0,797(+98,25) \\
0,875(+117,66) \\
0,907(+125,62) \\
0,916(+127,86) \\
0,835(+107,71) \\
0,780(+94,02) \\
0,755(+87,81) \\
\text { (b) }\end{array}$ & 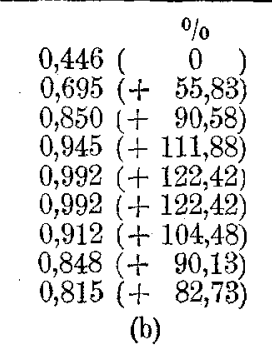 & 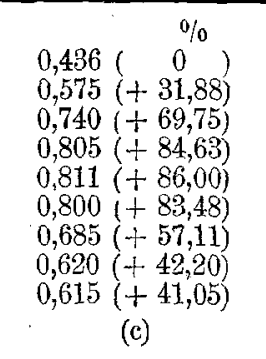 \\
\hline $\begin{array}{l}\text { Stunden in } \\
\text { der Lösung }\end{array}$ & $\begin{array}{c}100 \mathrm{ccm} 1 / 2 \mathrm{~m} . \\
\text { Harnstoff } \\
+10 \mathrm{ccm} 1 / 10 \mathrm{n} . \\
\mathrm{HCl}\end{array}$ & $\begin{array}{c}100 \mathrm{ccm} 1 / 2 \mathrm{~m} . \\
\text { Rohrzucker }+ \\
10 \mathrm{ccm} 1 / 10 \mathrm{n} . \mathrm{HCl}\end{array}$ & $\begin{array}{c}100 \mathrm{~cm} \mathrm{H}_{2} \mathrm{O}+ \\
10 \mathrm{ccm} 1 / 10 \mathrm{n} . \mathrm{HCl}\end{array}$ \\
\hline $\begin{array}{c}0 \\
0,33 \\
1,52 \\
3,25 \\
6,15 \\
7,40 \\
15,40 \\
30,55 \\
54,10\end{array}$ & $\begin{array}{c}0,0 \\
0,460\left(\begin{array}{c}0 \\
0\end{array}\right) \\
0,612(+38,03) \\
0,802(+74,34) \\
0,865(+87,82) \\
0,903(+96,30) \\
0,907(+97,17) \\
0,825(+79,34) \\
0,750(+63,04) \\
0,723(+57,17) \\
\text { c) }\end{array}$ & 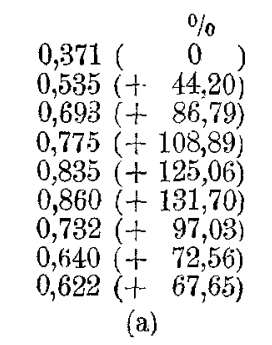 & $\begin{array}{c}\% \\
0,382\left(\begin{array}{c}0 \\
0\end{array}\right) \\
0,651(+70,41) \\
0,830(+117,28) \\
0,905(+163,09) \\
0,865(+126,43) \\
0,822(+115,17) \\
0,740(+93,45) \\
0,693(+81,41) \\
0,670 \text { (+ } 75,65) \\
\text { (a) }\end{array}$ \\
\hline
\end{tabular}

Zum Schluss möchte ich Herrn Dr. R. Benzinger, der mir freundlichst die Übersetzung durchgesehen hat, meinen verbindlichsten Dank aussprechen. 